TRANSACTIONS OF THE

AMERICAN MATHEMATICAL SOCIETY

Volume 358, Number 12, December 2006, Pages 5401-5423

S $0002-9947(06) 04037-2$

Article electronically published on July 20, 2006

\title{
HILBERT FUNCTIONS OF POINTS ON SCHUBERT VARIETIES IN THE SYMPLECTIC GRASSMANNIAN
}

\author{
SUDHIR R. GHORPADE AND K. N. RAGHAVAN
}

\begin{abstract}
We give an explicit combinatorial description of the multiplicity as well as the Hilbert function of the tangent cone at any point on a Schubert variety in the symplectic Grassmannian.
\end{abstract}

\section{INTRODUCTION}

Let $G$ be a semisimple algebraic group over an algebraically closed field $k$ and let $P$ be a parabolic subgroup of $G$. Fix a Borel subgroup $B$ of $G$ and a maximal torus $T$ in $G$ such that $T \subset B \subset P$. Now, $G / P$ is a projective variety and it has a distinguished class of subvarieties, known as Schubert varieties (in $G / P$ ); these are indexed by a set $W^{P}$, which corresponds to the $T$-fixed points of $G / P$ for the action given by left multiplication. Given $w \in W^{P}$, we denote by $e^{w}$ the corresponding $T$-fixed point, and by $X_{w}$ the corresponding Schubert variety. In fact, $X_{w}$ is the closure of the $B$-orbit $B e^{w}$, and can be decomposed as the union of 'smaller' $B$ orbits $B e^{v}$, where $v$ ranges over elements of $W^{P}$ satisfying $v \leq w$; here, $\leq$ is a certain partial order, called the Bruhat-Chevalley order.

The study of Schubert varieties, in general, and the singularities of Schubert varieties, in particular, has been an active and vibrant area of research in the past three decades. It may suffice to cite the recent monograph [3] by Billey and Lakshmibai, which surveys many known results and can also be a useful reference for the background material. Among the basic questions, insofar as the singularities of Schubert varieties are concerned, are the following: (1) which points are singular? (2) what is the multiplicity at a (singular) point? and (3) what is the Hilbert function (of the tangent cone) at a (singular) point? It may be noted that these questions are in an ascending order of generality since the singular points are those of multiplicity $>1$, and the Hilbert function determines the Hilbert polynomial whose (normalized) leading coefficient gives the multiplicity. Also note that, thanks to the $B$-orbit decomposition, it suffices to look only at the points $e^{v}$ in $X_{w}$ where $v \leq w$.

The singular loci of Schubert varieties are fairly well understood, thanks to the works of several mathematicians (see [3] for details). Recursive formulas for the multiplicity and for the Hilbert function in the case of minuscule $G / P$ and also in the case of symplectic Grassmannian were obtained by Lakshmibai and Weyman 22 in 1990. The singularities of Schubert varieties in the symplectic Grassmannian have also been studied by Brion and Polo [2] who determine the multiplicity at

Received by the editors September 20, 2004.

2000 Mathematics Subject Classification. Primary 14M15, 13F50, 13A30.

(c)2006 American Mathematical Society Reverts to public domain 28 years from publication 
'generic' singular points. More explicit results seem to be available in the special case when $G=\mathrm{SL}_{n}=\mathrm{SL}(V)$, where $V$ is an $n$-dimensional vector space over $k$ and $P=P_{d}$ the maximal parabolic subgroup given by those $g \in \mathrm{SL}(V)$ which stabilize a (fixed) $d$-dimensional subspace of $V$. Assume for a moment that we are in this case. Thus $G / P$ is the Grassmannian $G_{d}(V)$ and $W^{P}$ may be viewed as the set $I(d, n)$ of subsets of $\{1, \ldots, n\}$ of cardinality $d$. Let $\epsilon$ denote the element $\{1, \ldots, d\}$ of $I(d, n)$. An explicit closed-form formula for the multiplicity $m_{\epsilon}(w)$ of $X_{w}$ at $e^{\epsilon}$ was given by Lakshmibai and Weyman 22 in their 1990 paper. Recently, an explicit description for the Hilbert function of $X_{w}$ at $e^{\epsilon}$ was given by Kreiman and Lakshmibai [18. Further, in the case of arbitrary $v$, Kreiman and Lakshmibai formulated two conjectures which give an explicit combinatorial description for (i) the Hilbert function of $X_{w}$ at $e^{v}$ and (ii) the multiplicity $m_{\epsilon}(w)$ of $X_{w}$ at $e^{v}$. Subsequently, both conjectures were proved by Kodiyalam and Raghavan [14, and independently by Kreiman [17] (see also [19]). Both [14] and [17] also obtain a reformulation of the main result in terms of Gröbner bases.

In this paper, we consider the problem of determining the multiplicity as well as the Hilbert function of Schubert varieties in the symplectic Grassmannian $G / P$, where $G$ is the symplectic group $\mathrm{Sp}_{2 d}$ and $P$ is a maximal parabolic subgroup $P_{d}$ of $G$. Alternatively, $G / P$ is the Grassmannian of maximal isotropic subspaces (see Section 2 for details). For Schubert varieties in symplectic Grassmannians, we give an explicit combinatorial description for the multiplicity, and more generally, the Hilbert function at any $e^{v}$ in $X_{w}$. In effect, we formulate and prove the two conjectures of Kreiman and Lakshmibai in this case. Moreover, as in 14, we obtain a reformulation of the main result in terms of Gröbner bases. A precise statement of our main result is given in Section 2 ,

A key ingredient in our proof is the Standard Monomial Theory for symplectic Grassmannians and its Schubert subvarieties, as developed by De Concini [6] and by Lakshmibai, Musili and Seshadri 20. This allows us to translate the problem from geometry to combinatorics. This translation - although we derive it afresh in Section 3 below for the sake of clarity, completeness, and readability - is already there in Lakshmibai-Weyman 22. Our main job then is the solution of the resulting combinatorial problem. Roughly speaking, it amounts to showing that the combinatorial constructions of [14] behave well with respect to a certain involution. This is carried out in Sections 4 and 5. Moreover, as in 18 and 14, we obtain an interpretation of the multiplicity as the number of certain nonintersecting lattice paths, and this is described in Section 6 .

The interpretation of the multiplicity in terms of nonintersecting lattice paths may be viewed as an analogue of the results of Krattenthaler [15, 16. To review the latter, we note that in the case of the classical Grassmannian, Rosenthal and Zelevinsky [26] obtained in 1998 a closed-form formula for $m_{v}(w)$ for arbitrary $v$ and $w$, using the recursive formula of Lakshmibai and Weyman 22. The lattice path interpretation was used by Krattenthaler [15 to explain the relationship between the Rosenthal-Zelevinsky formula and the Lakshmibai-Weyman formula for $m_{\epsilon}(w)$. Further, Krattenthaler [16] also used the lattice path interpretation to prove the Multiplicity Conjecture of Kreiman and Lakshmibai.

We now attempt to outline a connection of Schubert varieties, in general, and the results of this paper, in particular, to a class of affine varieties, broadly known as determinantal varieties. For the last two decades, the study of determinantal 
varieties has proceeded almost in parallel to the study of Schubert varieties, but often as an independent pursuit. In particular, explicit formulas for the multiplicity and the Hilbert function for various classes of determinantal varieties have been obtained. The relation between determinantal varieties and Schubert varieties is best explained in the case of Grassmannian $G_{d}(V)=S L_{n} / P$. We have the wellknown Plücker embedding of $G_{d}(V)$ in $\mathbb{P}\left(\bigwedge^{d} V\right)$, and for each $v \in W^{P}$, there is a basic affine open set $\mathbb{A}_{v}$ containing $e^{v}$. In the case $v=\epsilon$, the intersections $X_{w} \cap \mathbb{A}_{v}$ are affine varieties, which are precisely the varieties defined by an ideal 'cogenerated' by a minor of a generic matrix; in other words, these are exactly the determinantal varieties studied by Abhyankar [1, and later by Herzog-Trung [12, Conca-Herzog [5], and others (see, e.g., 9, 10]). Moreover, this affine variety is a cone, and hence it coincides with the tangent cone to $X_{w}$ at $e^{\epsilon}$. Thus, in retrospect, the multiplicity formula of Lakshmibai-Weyman and the Hilbert function result of Kreiman-Lakshmibai could have been deduced from the work of Abhyankar [1] and others. On the other hand, for arbitrary $v$, the varieties $X_{w} \cap \mathbb{A}_{v}$ are not so well understood, and as far as we know, there is no analogue in the literature on determinantal varieties of the results in [14. As remarked in 14, the recent work of Knutson and Miller [13] considers a class of determinantal varieties more general than those cogenerated by a minor, but it is not clear if the varieties $X_{w} \cap \mathbb{A}_{v}$ belong to this class when $v \neq \epsilon$.

In a similar vein, considering affine patches of Schubert varieties in the symplectic Grassmannian, when $v=\epsilon$, leads to varieties given by ideals cogenerated by a minor of a generic symmetric matrix. Thus in this special case, the results obtained in this paper could be compared with those of Conca [4 on symmetric determinantal varieties. Likewise, one could take up the case of orthogonal Grassmannians (although we do not do this here), and the results thus obtained ought to be compared with those of Ghorpade and Krattenthaler [1] on pfaffian varieties. In either of these cases, for an arbitrary $v$, the varieties $X_{w} \cap \mathbb{A}_{v}$ do not seem to correspond to any of the known classes of varieties defined by the minors of a generic symmetric matrix or the pfaffians of a generic skew-symmetric matrix.

There is a yet another related, but independent, body of work on degeneracy loci. Formulas for the fundamental classes of degeneracy loci of maps of vector bundles give rise to multiplicity formulas for determinantal and pfaffian varieties. For a detailed explanation, we refer to the appendix in [11 and the books of FultonPragacz [8] and Manivel [25]. While some of the results on multiplicity could be deduced from the corresponding results on degeneracy loci, it does not seem likely that the latter impinge on the determination of Hilbert functions.

\section{THE THEOREM}

The main results of this paper are stated in Theorem 2.1 and Corollary 2.2 below. But first, we fix some notation and terminology to be used in the rest of this paper and briefly review some preliminary notions and results.

Given any nonnegative integer $n$, we denote by $[n]$ the set $\{1, \ldots, n\}$. The cardinality of a finite set $v$ will be denoted by $|v|$. Given positive integers $r$ and $n$ with $r \leq n$, we denote by $I(r, n)$ the set of all subsets of $[n]$ of cardinality $r$. An element $v$ of $I(r, n)$ may be written as $v=\left(v_{1}, \ldots, v_{r}\right)$, where $1 \leq v_{1}<\ldots<v_{r} \leq n$ and $v=\left\{v_{1}, \ldots, v_{r}\right\}$. Given any $v=\left(v_{1}, \ldots, v_{r}\right)$ and $w=\left(w_{1}, \ldots, w_{r}\right)$ in $I(r, n)$, we say $v \leq w$ if $v_{1} \leq w_{1}, \ldots, v_{r} \leq w_{r}$. Clearly, $\leq$ defines a partial order on $I(r, n)$. 
A positive integer $d$ will be kept fixed throughout this paper. For $j \in[2 d]$, set $j^{*}:=2 d+1-j$. Let $I(d)$ denote the set of subsets $v$ of $[2 d]$ with the property that exactly one of $j, j^{*}$ belongs to $v$ for every $j \in[d]$. Note that $I(d)$ is a subset of $I(d, 2 d)$. In particular, we have the partial order $\leq$ on $I(d)$ induced from $I(d, 2 d)$. We denote by $\epsilon$ the element $[d]=(1, \ldots, d)$ of $I(d)$.

Fix a vector space $V$ of dimension $2 d$ over an algebraically closed field of arbitrary characteristic. Fix a nondegenerate skew-symmetric bilinear form $\langle$,$\rangle on V$. Fix a basis $e_{1}, \ldots, e_{2 d}$ of $V$ such that

$$
\left\langle e_{i}, e_{j}\right\rangle=\left\{\begin{aligned}
1 & \text { if } i=j^{*} \text { and } i<j \\
-1 & \text { if } i=j^{*} \text { and } i>j \\
0 & \text { otherwise. }
\end{aligned}\right.
$$

A linear subspace of $V$ is said to be isotropic if the form $\langle$,$\rangle vanishes identically$ on it. It is well known that an isotropic subspace of $V$ has dimension at most $d$ and every isotropic subspace is contained in one of dimension $d$. Denote by $G_{d}(V)$ the Grassmannian of $d$-dimensional subspaces of $V$ and by $\mathfrak{M}_{d}(V)$ the set of all $d$-dimensional isotropic subspaces of $V$. Then $\mathfrak{M}_{d}(V)$ is a closed subvariety of $G_{d}(V)$ and is called the variety of maximal isotropic subspaces or the symplectic Grassmannian.

The group $\operatorname{Sp}(V)$ of linear automorphisms of $V$ preserving $\langle$,$\rangle acts transitively$ on $\mathfrak{M}_{d}(V)$ - this follows from Witt's theorem that an isometry between subspaces can be lifted to one of the whole vector space. Using this, $\mathfrak{M}_{d}(V)$ can be identified as the quotient of $\operatorname{Sp}(V)$ by the stabilizer of any point (for example, the span of $\left.e_{1}, \ldots, e_{d}\right)$. The elements of $\operatorname{Sp}(V)$ that are diagonal with respect to the basis $e_{1}$, $\ldots, e_{2 d}$ form a maximal torus $T$ of $\operatorname{Sp}(V)$. Similarly the elements of $\operatorname{Sp}(V)$ that are upper triangular with respect to $e_{1}, \ldots, e_{2 d}$ form a Borel subgroup $B$ of $\operatorname{Sp}(V)$ a linear transformation is upper triangular with respect to $e_{1}, \ldots, e_{2 d}$ if for each $j \in[2 d]$, the image of $e_{j}$ under the transformation is a linear combination of $e_{i}$ with $i \leq j$.

The $T$-fixed points of $\mathfrak{M}_{d}(V)$ are parametrized by $I(d)$ : for $v=\left(v_{1}, \ldots, v_{d}\right)$ in $I(d)$, the corresponding $T$-fixed point, denoted by $e^{v}$, is the span of $e_{v_{1}}, \ldots, e_{v_{d}}$. These points lie in different $B$-orbits, and the union of their $B$-orbits is all of $\mathfrak{M}_{d}(V)$. A Schubert variety in $\mathfrak{M}_{d}(V)$ is by definition the closure of such a $B$-orbit (with the reduced scheme structure). Schubert varieties are thus indexed by the $T$-fixed points and so in turn by $I(d)$. Given $w$ in $I(d)$, we denote by $X_{w}$ the closure of the $B$-orbit of the $T$-fixed point $e^{w}$. We have the $B$-orbit decomposition:

$$
X_{w}=\coprod_{v \leq w} B e^{v} .
$$

We are interested in the local rings of various points on a Schubert variety $X_{w}$. In view of the above $B$-orbit decomposition, it is enough to focus our attention on the $T$-fixed points contained in $X_{w}$, that is, the points $e^{v}$ for $v \leq w$.

For the rest of this section, fix elements $v, w$ of $I(d)$ with $v \leq w$. Define

$$
\mathfrak{R}^{v}:=\left\{(r, c) \in[2 d] \backslash v \times v: r \leq c^{*}\right\} \quad \text { and } \quad \mathfrak{N}^{v}:=\{(r, c) \in[2 d] \backslash v \times v: r>c\} .
$$

We will be considering "multisets" on $\mathfrak{R}^{v}$ and $\mathfrak{N}^{v}$. By a multiset on a finite set $S$ we mean a collection of elements of $S$ in which repetitions are allowed and kept account of. Multisets on $S$ can be thought of as monomials in the variables corresponding to the elements of $S$. The cardinality of a multiset is the number of 
elements in it, counting repetitions, or equivalently, the degree of the corresponding monomial. The union of multisets is the product of the corresponding monomials. The intersection of a multiset with a subset is again easily described in terms of monomials: set equal to 1 those variables that do not belong to the subset.

Given any $\beta_{1}=\left(r_{1}, c_{1}\right), \beta_{2}=\left(r_{2}, c_{2}\right)$ in $\mathfrak{N}^{v}$, we say that $\beta_{1}>\beta_{2}$ if $r_{1}>r_{2}$ and $c_{1}<c_{2}$. A sequence $\beta_{1}>\ldots>\beta_{t}$ of elements of $\mathfrak{N}^{v}$ is called a $v$-chain. Given a $v$-chain $\beta_{1}=\left(r_{1}, c_{1}\right)>\ldots>\beta_{t}=\left(r_{t}, c_{t}\right)$, we define

$$
s_{\beta_{1}} \cdots s_{\beta_{t}} v:=\left(\left\{v_{1}, \ldots, v_{d}\right\} \backslash\left\{c_{1}, \ldots, c_{t}\right\}\right) \cup\left\{r_{1}, \ldots, r_{t}\right\},
$$

and note that this is an element of $I(d)$. In case the $v$-chain is empty, this element is just $v$. We say that $w$ dominates the $v$-chain $\beta_{1}>\ldots>\beta_{t}$ if $w \geq s_{\beta_{1}} \cdots s_{\beta_{t}} v$.

Let $\mathfrak{S}$ be a monomial on $\mathfrak{R}^{v}$. By a $v$-chain in $\mathfrak{S}$ we mean a sequence $\beta_{1}>\ldots>\beta_{t}$ of elements of $\mathfrak{S} \cap \mathfrak{N}^{v}$. We say that $w$ dominates $\mathfrak{S}$ if $w$ dominates every $v$-chain in $\mathfrak{S}$.

Let $S_{w}^{v}$ denote the set of $w$-dominated monomials on $\Re^{v}$, and let $S_{w}^{v}(m)$ be the set of such monomials of degree $m$.

We can now state our theorem.

Theorem 2.1. Let $v, w$ be elements of $I(d)$ with $v \leq w$. Let $X_{w}$ be the Schubert variety corresponding to $w, e^{v}$ the $T$-fixed point corresponding to $v$, and $R$ the coordinate ring of the tangent cone to $X_{w}$ at the point $e^{v}$ (that is, the associated graded ring $\operatorname{gr}_{M}\left(\mathcal{O}_{X_{w}, e^{v}}\right)$ of the local ring $\mathcal{O}_{X_{w}, e^{v}}$ of $X_{w}$ at the point $e^{v}$ with respect to its maximal ideal $M)$. Then the dimension as a vector space of the $m^{\text {th }}$ graded piece $R(m)$ of $R$ equals the cardinality of $S_{w}^{v}(m)$, where $S_{w}^{v}(m)$ is as defined above.

The proof of the theorem occupies Sections 3, 4, and 5, For now let us note the following easy consequence.

Corollary 2.2. With notation as in Theorem 2.1 above, the multiplicity of $R$ equals the number of square-free $w$-dominated monomials on $\mathfrak{R}^{v}$ of maximum cardinality.

Proof. The proof of the corresponding corollary in [14] works verbatim here.

\section{Reduction to COMbinatorics}

Let $\mathfrak{M}_{d}(V) \subseteq G_{d}(V) \hookrightarrow \mathbb{P}\left(\wedge^{d} V\right)$ be the Plücker embedding. The homogeneous coordinate rings of $\mathfrak{M}_{d}(V)$ and its Schubert subvarieties in this embedding have been described by De Concini [6] and Lakshmibai, Musili, and Seshadri [20]. We will use their results to reduce the proof of Theorem 2.1 to combinatorics. Our primary reference will be [6] - its language and approach suit our purpose well.

For $\theta$ in $I(d, 2 d)$, let $p_{\theta}$ denote the corresponding Plücker coordinate. Consider the affine patch $\mathbb{A}$ of $\mathbb{P}\left(\bigwedge^{d} V\right)$ given by $p_{\epsilon}=1$. The intersection $\mathbb{A} \cap G_{d, 2 d}$ of this patch with the Grassmannian is an affine space. Indeed the $d$-plane corresponding to an arbitrary point $z$ of $\mathbb{A} \cap G_{d, 2 d}$ has a basis consisting of column vectors of a matrix of the form

$$
B=\left(\begin{array}{l}
I \\
A
\end{array}\right),
$$

where $I$ is the identity matrix of size $d \times d$ and $A$ is an arbitrary matrix of size $d \times d$. The association $z \mapsto A$ is bijective. The restriction of a Plücker coordinate $p_{\theta}$ to $\mathbb{A} \cap G_{d, 2 d}$ is given by the determinant of a submatrix of size $d \times d$ of $B$, the entries of $\theta$ determining the rows to be chosen from $B$ to form the submatrix. 
As can be readily verified, a point $z$ of $\mathbb{A} \cap G_{d, 2 d}$ belongs to $\mathfrak{M}_{d}(V)$ if and only if the corresponding matrix $A=\left(a_{i j}\right)$ is symmetric with respect to the anti-diagonal: $a_{i j}=a_{j^{*} i^{*}}$, where the columns and rows of $A$ are numbered $1, \ldots, d$ and $d+1, \ldots, 2 d$ respectively, and $r^{*}:=2 d-r+1$ for $r \in[2 d]$. For example, if $d=4$, then a matrix that is symmetric with respect to the anti-diagonal looks like this:

$$
\left(\begin{array}{llll}
d & c & b & a \\
g & f & e & b \\
i & h & f & c \\
j & i & g & d
\end{array}\right) .
$$

Notation 3.1. For $u=\left(u_{1}, \ldots, u_{d}\right)$ in $I(d, 2 d)$, set $u^{*}:=\left(u_{d}^{*}, \ldots, u_{1}^{*}\right)$. The association $u \mapsto u^{*}$ is an order reversing involution of $I(d, 2 d)$. There is another order reversing involution on $I(d, 2 d)$, namely $u \mapsto[2 d] \backslash u$. These two involutions commute with each other. Composing them, we obtain an order preserving involution on $I(d, 2 d): u \mapsto u^{\#}:=[2 d] \backslash u^{*}$. Note that $u \in I(d)$ if and only if $u=u^{\#}$.

Lemma 3.2. Given any $\theta \in I(d, 2 d)$, we have $p_{\theta}= \pm p_{\theta \#}$ on $\mathfrak{M}_{d}(V)$.

Proof. Since $\mathfrak{M}_{d}(V)$ is irreducible and its intersection $\mathbb{A} \cap \mathfrak{M}_{d}(V)$ with the affine patch is nonempty, it is enough to check that $p_{\theta}= \pm p_{\theta \#}$ on $\mathbb{A} \cap \mathfrak{M}_{d}(V)$, and this follows from the symmetry property of the matrix $A$ just mentioned.

The sign factor \pm in Lemma 3.2 can be determined explicitly (although that will play no part in the sequel): it equals $(-1)^{t}$, where $t=\left(\theta_{1}-1\right)+\cdots+\left(\theta_{k}-k\right)+$ $\left(\theta_{k+1}-(d+1)\right)+\cdots+\left(\theta_{d}-(2 d-k)\right)$ and $k=|[d] \cap \theta|$. The relations $p_{\theta}= \pm p_{\theta \#}$ do not span the space of all linear relations among the $p_{\theta}$; see Example 3.5 below. In order to describe a nice parametrizing set for a basis for the space of linear forms in the homogeneous coordinate ring of $\mathfrak{M}_{d}(V)$-in fact for describing bases for spaces of forms of any given degree - we make the following definition.

Definition 3.3.1 The $\epsilon$-degree of an element $x$ of $I(d)$ is the cardinality of $x \backslash[d]$ or equivalently that of $[d] \backslash x$. More generally, given any $v \in I(d)$, the $v$-degree of an element $x$ of $I(d)$ is the cardinality of $x \backslash v$ or equivalently that of $v \backslash x$. An ordered pair $\mathfrak{w}=(x, y)$ of elements of $I(d)$ is called an admissible pair if $x \geq y$ and the $\epsilon$-degrees of $x$ and $y$ are equal. We refer to $x$ and $y$ as the top and the bottom of $\mathfrak{w}$ and write $\mathfrak{t o p}(\mathfrak{w})$ for $x$ and $\mathfrak{b o t}(\mathfrak{w})$ for $y$.

Given any admissible pairs $\mathfrak{w}=(x, y)$ and $\mathfrak{w}^{\prime}=\left(x^{\prime}, y^{\prime}\right)$, we say $\mathfrak{w} \geq \mathfrak{w}^{\prime}$ if $y \geq x^{\prime}$, that is, if $x \geq y \geq x^{\prime} \geq y^{\prime}$. An ordered sequence $\left(\mathfrak{w}_{1}, \ldots, \mathfrak{w}_{t}\right)$ of admissible pairs is called a standard tableau if $\mathfrak{w}_{i} \geq \mathfrak{w}_{i+1}$ for $1 \leq i<t$. We often write $\mathfrak{w}_{1} \geq \ldots \geq \mathfrak{w}_{t}$ to denote the standard tableau $\left(\mathfrak{w}_{1}, \ldots, \mathfrak{w}_{t}\right)$. Given any $w \in I(d)$, we say that a standard tableau $\mathfrak{w}_{1} \geq \ldots \geq \mathfrak{w}_{t}$ is $w$-dominated if $w \geq \mathfrak{t o p}\left(\mathfrak{w}_{1}\right)$.

\footnotetext{
${ }^{1}$ Admissible pairs as defined here are a special case of the admissible minors of De Concini [6]: we are only considering the case $k=r$ in his notation. Our standard tableaux are his standard symplectic tableaux, but here again we are only considering the special case $k=r$.

The original definition of admissible pairs by Lakshmibai-Musili-Seshadri [20, Part A, §3] is in the more general context of a quotient by a maximal parabolic subgroup of classical type of a semisimple algebraic group. The realization of the importance of admissible pairs was a key step in their development of standard monomial theory. The definition given here is equivalent, in the special case being considered, to theirs.

In Littelmann's language of paths [23, 24], an admissible pair is just an $L-S$ path of shape a fundamental weight of classical type.
} 
Proposition 3.4. There is a bijective map $(x, y) \mapsto(\theta, \tau)$ from the set of admissible pairs $(x, y)$ onto the set of ordered pairs $(\theta, \tau)$ of elements of $I(d, 2 d)$ satisfying

$$
\tau=\theta^{\#} \quad \text { and } \quad \theta \cap[d] \geq \theta^{\#} \cap[d] .
$$

Proof. Let $[d]^{\mathrm{c}}:=[2 d] \backslash[d]=\{d+1, \ldots, 2 d\}$. Given an admissible pair $(x, y)$, set

$$
\theta=(x \cap[d]) \cup\left(y \cap[d]^{\mathrm{c}}\right) \quad \text { and } \quad \tau=(y \cap[d]) \cup\left(x \cap[d]^{\mathrm{c}}\right) .
$$

That $(\theta, \tau)$ satisfies the two conditions is readily verified. For the map in the other direction, set

$$
x=(\theta \cap[d]) \cup\left(\tau \cap[d]^{\mathrm{c}}\right) \quad \text { and } \quad y=(\tau \cap[d]) \cup\left(\theta \cap[d]^{\mathrm{c}}\right) .
$$

It is easy to verify that $(x, y)$ is an admissible pair.

Example 3.5. The relations $p_{\theta}= \pm p_{\theta}$ do not span the linear space of relations although [22, page 198, item (b)] seems to claim just that. For example, let

$$
d=4, \quad \theta_{1}=(1,2,7,8), \quad \theta_{2}=(1,4,5,8) \quad \text { and } \quad \theta_{3}=(1,3,6,8) .
$$

Then

$$
\theta_{1}^{\#}=(3,4,5,6), \quad \theta_{2}^{\#}=(2,3,6,7) \quad \text { and } \quad \theta_{3}^{\#}=(2,4,5,7) .
$$

From the form displayed above of a matrix that is symmetric with respect to the anti-diagonal, we get

$$
p_{\theta_{1}}=p_{\theta_{1}^{\#}}=d f-c g, \quad p_{\theta_{2}}=p_{\theta_{2}^{\#}}=c g-b i \quad \text { and } \quad p_{\theta_{3}}=p_{\theta_{3}^{\#}}=b i-d f,
$$

and consequently, $p_{\theta_{1}}+p_{\theta_{2}}+p_{\theta_{3}}=0$.

Again, contrary to what is claimed in [22, item (10), page 199], the association of the previous proposition is not a bijection if the second condition is dropped. In the case $d=4$ for example there are 42 admissible pairs $(x, y)$ and 43 pairs $\left(\theta, \theta^{\#}\right)$. If we try the procedure in the proof above for recovering $(x, y)$ on an arbitrary $\left(\theta, \theta^{\#}\right)$, the two resulting elements may not be comparable. Taking for example $\theta=(2,3,6,7), \theta^{\#}=(1,4,5,8)$, we recover $(2,3,5,8)$ and $(1,4,6,7)$.

Definition 3.6. Given an admissible pair $\mathfrak{w}=(x, y)$, we define the associated Plücker coordinate $p_{\mathfrak{w}}$ to be $p_{\theta}$, where $(x, y) \mapsto\left(\theta, \theta^{\#}\right)$ by the association of Proposition 3.4. Observe that $p_{\theta}= \pm p_{\theta \#}$ by Lemma 3.2. If $x=y$ we sometimes write $p_{x}$ for $p_{(x, x)}$. To formal products of admissible pairs we associate the product of the associated Plücker coordinates. In particular, to a standard tableau $\mathfrak{w}_{1} \geq \ldots \geq \mathfrak{w}_{t}$ we associate $p_{\mathfrak{w}_{1}} \cdots p_{\mathfrak{w}_{t}}$. Such monomials associated to standard tableaux are called standard monomials. Given any $w$ in $I(d)$, we say that a standard monomial is $w$-dominated if the corresponding standard tableau is $w$-dominated.

We can now state the main theorem of standard monomial theory for $\mathfrak{M}_{d}(V)$ and its Schubert subvarieties.

Theorem 3.7 (De Concini, Lakshmibai-Musili-Seshadri). Standard monomials $p_{\mathfrak{w}_{1}} \cdots p_{\mathfrak{w}_{t}}$ of degree $r$ form a basis for the space of forms of degree $r$ in the homogeneous coordinate ring of $\mathfrak{M}_{d}(V)$ in the Plücker embedding. More generally, given any $w \in I(d)$, the $w$-dominated standard monomials of degree $r$ form a basis for the space of forms of degree $r$ in the homogeneous coordinate ring of the Schubert subvariety $X(w)$ of $\mathfrak{M}_{d}(V)$. 
Proof. The linear independence of the $w$-dominated standard monomials in the homogeneous coordinate ring of $X_{w}$ is proved in [6, Lemma 3.5].2 That all standard monomials span the homogeneous coordinate ring of $\mathfrak{M}_{d}(V)$ (and so also that of $X_{w}$ ) is the content of [6, Theorem 2.4]. On the other hand, as is easy to see, $p_{\vartheta}$ vanishes on $X_{w}$ unless $w \geq \mathfrak{t o p}(\vartheta)$, and so the standard monomials that are not $w$-dominated vanish on $X_{w}$.

From the above theorem we now deduce a basis for the coordinate ring for an affine patch of a Schubert subvariety in $\mathfrak{M}_{d}(V)$.

Definition 3.8. Given any $v \in I(d)$, we say that a standard tableau $\mathfrak{w}_{1} \geq \ldots \geq \mathfrak{w}_{t}$ is $v$-compatible if for each $\mathfrak{w}_{i}$, either $v \geq \mathfrak{t o p}\left(\mathfrak{w}_{i}\right)$ or $\mathfrak{b o t}\left(\mathfrak{w}_{i}\right) \geq v$, and $\mathfrak{w}_{i} \neq(v, v)$. A standard monomial is $v$-compatible if the corresponding standard tableau is $v$ compatible. Given $v$ and $w$ in $I(d)$, we denote by $\mathrm{SM}_{w}^{v}$ the set of $w$-dominated $v$-compatible standard tableaux.

Fix elements $v \leq w$ of $I(d)$ so that the point $e^{v}$ belongs to the Schubert variety $X(w)$. Let $\mathbb{A}_{v}$ denote the affine patch of $\mathbb{P}\left(\bigwedge^{d} V\right)$ given by $p_{v} \neq 0$ and set

$$
Y_{w}^{v}:=X(w) \cap \mathbb{A}_{v} .
$$

The point $e^{v}$ is the origin of the affine space $\mathbb{A}_{v}$.

The functions $f_{\mathfrak{w}}=p_{\mathfrak{w}} / p_{v}$, where $\mathfrak{w}$ varies over admissible pairs, provide a set of coordinate functions on $\mathbb{A}_{v}$. The coordinate ring $k\left[Y_{w}^{v}\right]$ of $Y_{w}^{v}$ is a quotient of the polynomial ring $k\left[f_{\mathfrak{w}}\right]$, where $k$ is the underlying field.

Proposition 3.9. As $\mathfrak{w}_{1} \geq \ldots \geq \mathfrak{w}_{t}$ runs over the set $S M_{w}^{v}$ of $w$-dominated $v$-compatible standard tableaux, the elements $f_{\mathfrak{w}_{1}} \cdots f_{\mathfrak{w}_{t}}$ form a basis for the coordinate ring $k\left[Y_{w}^{v}\right]$ of the affine patch $Y_{w}^{v}=X_{w} \cap \mathbb{A}^{v}$ of the Schubert variety $X_{w}$.

Proof. The proof is similar to the proof of Proposition 3.1 of [14]. First consider any linear dependence relation among the $f_{\mathfrak{w}_{1}} \cdots f_{\mathfrak{w}_{t}}$. Replacing $f_{\mathfrak{w}}$ by $p_{\mathfrak{w}}$ and "homogenizing" by $p_{v}$ yields a linear dependence relation among the $w$-dominated standard monomials $p_{\vartheta_{1}} \cdots p_{\vartheta_{s}}$ restricted to $X_{w}$, and so the original relation must only have been the trivial one, for by Theorem 3.7 the $p_{\vartheta_{1}} \cdots p_{\vartheta_{s}}$ are linearly independent.

To prove that the $f_{\mathfrak{w}_{1}} \cdots f_{\mathfrak{w}_{t}}$ span $k\left[Y_{w}^{v}\right]$ as a vector space, we need to look at not only the corresponding statement for the Plücker coordinates but also the proof of that statement. What is immediate from the corresponding statement for the Plücker coordinates is that $f_{\vartheta_{1}} \ldots f_{\vartheta_{s}}$ span $k\left[Y_{w}^{v}\right]$ as $\vartheta_{1} \geq \ldots \geq \vartheta_{s}$ varies over $w$-dominated standard monomials - the problem at hand is to show that the $v$-compatible ones among these are enough.

To an arbitrary monomial $p_{\vartheta_{1}} \cdots p_{\vartheta_{s}}$ in the Plücker coordinates, attach the following multiset of $\{1, \ldots, 2 d\}$ :

$$
\mathfrak{t o p}\left(\vartheta_{1}\right) \cup \mathfrak{b o t}\left(\vartheta_{1}\right) \cup \cdots \cup \mathfrak{t o p}\left(\vartheta_{s}\right) \cup \mathfrak{b o t}\left(\vartheta_{s}\right) .
$$

We claim that if $p_{\varphi_{1}} \cdots p_{\varphi_{s}}$ is a standard monomial that occurs with nonzero coefficient in the expression of $p_{\vartheta_{1}} \cdots p_{\vartheta_{s}}$ as a linear combination of standard monomials, the multiset attached to the two monomials are the same. The claim follows from the nature of the relations used in the proof in [6] of the spanning by the standard

\footnotetext{
${ }^{2}$ The Schubert varieties in 6] are orbits under the lower triangular Borel subgroup as opposed to our choice of upper triangular here, and that is why the domination is reversed in the statement of the lemma there.
} 
monomials 3 Now let $f_{\varphi_{1}} \cdots f_{\varphi_{s}}$ be an arbitrary monomial. Consider the expression as a linear combination of standard monomials for $p_{v}^{h} \cdot p_{\varphi_{1}} \cdots p_{\varphi_{s}}$, where $h$ is larger than $2 d s$. It follows from the claim that $p_{v}$ must occur in every monomial on the right. Dividing by $p_{v}^{h+s}$ we get an expression for $f_{\varphi_{1}} \cdots f_{\varphi_{s}}$ as a linear combination of $f_{\mathfrak{w}_{1}} \cdots f_{\mathfrak{w}_{t}}$ where $\mathfrak{w}_{1} \geq \ldots \geq \mathfrak{w}_{t}$ are $v$-compatible.

Given an admissible pair $\mathfrak{w}=(x, y)$, define the $v$-degree of $\mathfrak{w}$ by

$$
v \text {-degree }(\mathfrak{w}):=\frac{1}{2}(|x \backslash v|+|y \backslash v|) .
$$

An easy calculation using the fact that the $\epsilon$-degrees of $x$ and $y$ are equal gives the following:

$$
v \text {-degree }(\mathfrak{w})=|\theta \backslash v|=\left|\theta^{\#} \backslash v\right|,
$$

where $\left(\theta, \theta^{\#}\right)$ is the pair associated to $\mathfrak{w}=(x, y)$ by Proposition 3.4 above.

The affine patch $G_{d}(V) \cap \mathbb{A}_{v}$ of the Grassmannian is an affine space whose coordinate ring can be taken to be the polynomial ring in variables of the form $X_{r c}$, where $r$ and $c$ are numbers between 1 and $2 d$ such that $c$ belongs to $v$ and $r$ does not - this is easy to see and in any case explained in [14, §3]. It is readily seen that the ideal of the closed subvariety $\mathfrak{M}_{d}(V) \cap \mathbb{A}^{v}$ of $G_{d, 2 d} \cap \mathbb{A}_{v}$ is generated by elements of the form $X_{r c}-\epsilon X_{c^{*} r^{*}}$, where $\epsilon=-1$ if either $r>d$ and $c^{*}<d$ or $r<d$ and $c^{*}>d$, and $\epsilon=1$ otherwise. The affine patch $\mathfrak{M}_{d}(V) \cap \mathbb{A}^{v}$ of $\mathfrak{M}_{d}(V)$ is thus also an affine space whose coordinate ring can be taken to be the polynomial ring in variables of the form $X_{\beta}, \beta$ in $\mathfrak{R}^{v}$. Taking $d=5$ and $v=(3,4,6,9,10)$ for

\footnotetext{
${ }^{3}$ There are two types of relations used in the proof: those in equation (1.1) and those in Proposition 1.8 (all numbers as in [6]). To get the theorem about spanning by standard monomials in our situation, we only need to use special cases of these, and so let us first specialize.

In (1.1) we take $s=s^{\prime}$ and further let these equal our $d$ (our $d$ is De Concini's $r$ ); the latter halves of all minors are $1, \ldots, d$ for us, and so we write only the first half-a minor for us is therefore just an element $\theta$ of $I(d, 2 d)$; the right side $H$ is 0 in our case by the choice $s=d$. Equation (1.1) gets used in the following way: whenever we have a 'bad' product of two minors, it occurs as a term on the left side of an equation of type (1.1) where the other terms are 'not so bad'; the right side being 0 , this allows replacement of a 'bad' product by a linear combination of 'not so bad' ones. Observe the following: for each term on the left side, the multiset of $\{1, \ldots, 2 d\}$ that is the union of the indices of the two minors, is constant for all terms.

Let us denote a minor $\theta$ by the pair $\left(\theta, \theta^{\#}\right)$ rather than by just $\theta$. For each term $\theta \tau$ on the left side of (1.1) let us consider the multiset $\theta \cup \theta^{\#} \cup \tau \cup \tau^{\#}$; it follows from the observation in the last line of the previous paragraph that this multiset is constant for all terms.

We need Proposition 1.8 only in the case where $k$ is $d$ and $h_{1}, \ldots, h_{k}$ is $1, \ldots, d$ (we will omit writing the $\left.h_{1}, \ldots, h_{k}\right)$. Let $\theta$ be the element of $I(d, 2 d)$ that denotes the minor that is denoted $(\tilde{J} \cup \Gamma, \tilde{I} \cup \Gamma)$ in $[6](\tilde{J}, \tilde{I}, \Gamma$ are subsets of $\{1, \ldots, d\} ; \Gamma$ does not meet $\tilde{J} \cup \tilde{I}$; it is allowed that $\tilde{I} \cap \tilde{J}$ is nonempty). Write $M:=(\tilde{I} \cap \tilde{J}) \cup \Gamma, N:=\{1, \ldots, d\} \backslash(\tilde{I} \cup \tilde{J} \cup \Gamma), \hat{I}:=\tilde{I} \backslash \tilde{J}$, and $\hat{J}:=\tilde{J} \backslash \tilde{I}$. Then $\theta=\hat{I} \cup \hat{J}^{\star} \cup M \cup M^{\star}$ and $\theta^{\#}=\hat{I} \cup \hat{J}^{\star} \cup N \cup N^{*}$. In other words, the elements outside of $\hat{I} \cup \hat{J}^{\star}$ occur in $\theta \cup \theta^{\star}$ with multiplicity 2 , those of $\hat{I}^{\star} \cup \hat{J}$ with multiplicity 0 , and the rest of $\{1, \ldots, 2 d\}$ with multiplicity 1 . Now, the right side of the equation in Proposition 1.8 consists of minors of the form $(\tilde{J} \cup \bar{\Gamma}, \tilde{I} \cup \bar{\Gamma})$, where $\bar{\Gamma}$ has the same cardinality as $\Gamma$ and is contained in the complement of $\tilde{I} \cup \tilde{J} \cup \Gamma$. Since $\hat{I}$ and $\hat{J}$ remain invariant for all minors appearing in the equation, it follows that the multiset $\theta \cup \theta^{\#}$ is the same for all terms.

Finally, observe that if $\left(\theta, \theta^{\#}\right)$ corresponds to an admissible pair, say $(x, y)$, then $\theta \cup \theta^{\#}=$ $x \cup y$; see the proof of Proposition 3.4 above. Thus when we rewrite a monomial in the Plücker coordinates in terms of the minors of [6], use the relations of equation (1.1) and Proposition 1.8 as in the proof of Theorem 2.4 of [6] to express it as a linear combination of "standard symplectic tableaux", and then translate back to get a linear combination of standard monomials in our language, the multiset attached to any monomial on the right side is the same as the multiset attached to the original monomial.
} 
example, a general element of $\mathfrak{M}_{d}(V) \cap \mathbb{A}_{v}$ has a basis consisting of column vectors of a matrix of the following form-having a negative sign in front of all the variable entries in the first $d$ rows is just a convenient way of getting the signs right:

$$
\left(\begin{array}{ccccc}
-X_{13} & -X_{14} & -X_{16} & -X_{19} & -X_{1,10} \\
-X_{23} & -X_{24} & -X_{26} & -X_{29} & -X_{19} \\
1 & 0 & 0 & 0 & 0 \\
0 & 1 & 0 & 0 & 0 \\
-X_{53} & -X_{54} & -X_{56} & -X_{26} & -X_{16} \\
0 & 0 & 1 & 0 & 0 \\
X_{73} & X_{74} & X_{54} & X_{24} & X_{14} \\
X_{83} & X_{73} & X_{53} & X_{23} & X_{13} \\
0 & 0 & 0 & 1 & 0 \\
0 & 0 & 0 & 0 & 1
\end{array}\right) .
$$

The expression for $f_{\mathfrak{w}}:=p_{\mathfrak{w}} / p_{v}$ in terms of the $X_{\beta}, \beta \in \mathfrak{R}^{v}$, is obtained by taking the determinant of the submatrix of a matrix such as above obtained by choosing the rows given by the entries of $\theta$, where $\mathfrak{w} \mapsto\left(\theta, \theta^{\#}\right)$ is the association in Proposition 3.4. Thus it is a homogeneous polynomial of degree the $v$-degree of $\mathfrak{w}$. Since the ideal of the Schubert variety $X_{w}$ in the homogeneous coordinate ring of $\mathfrak{M}_{d}(V) \subseteq \mathbb{P}\left(\bigwedge^{d} V\right)$ is generated by the $p_{\mathfrak{w}}$ as $\mathfrak{w}=(x, y)$ varies over all admissible pairs such that $w \nsupseteq x \mathbb{4}^{4}$ it follows that the ideal of $Y_{w}^{v}:=X_{w} \cap \mathbb{A}^{v}$ in $\mathfrak{M}_{d}(V) \cap \mathbb{A}^{v}$ is generated by the same $f_{\mathfrak{w}}$. We are interested in the tangent cone to $X_{w}$ at $e^{v}$ (or what is the same, the tangent cone to $Y_{w}^{v}$ at the origin), and since $k\left[Y_{w}^{v}\right]$ is graded, its associated graded ring with respect to the maximal ideal corresponding to the origin is $k\left[Y_{w}^{v}\right]$ itself.

Proposition 3.9 tells us that the graded piece of $k\left[Y_{w}^{v}\right]$ is generated as a $k$-vector space by elements of $\mathrm{SM}_{w}^{v}$ of degree $m$, where the degree of a standard monomial $\mathfrak{w}_{1} \geq \ldots \geq \mathfrak{w}_{t}$ is defined to be the sum of the $v$-degrees of $\mathfrak{w}_{1}, \ldots, \mathfrak{w}_{t}$. To prove Theorem 2.1 it therefore suffices to prove that the $\operatorname{set}^{v} \operatorname{SM}_{w}^{v}(m)$ of $w$-dominated $v$-compatible standard monomials of degree $m$ is in bijection with $S_{w}^{v}(m)$.

\section{FURTHER REDUCTIONS}

In the last section we saw how Theorem 2.1 follows once it is shown that the combinatorially defined sets $\operatorname{SM}_{w}^{v}(m)$ and $S_{w}^{v}(m)$ are in bijection with each other. Now we make some further reductions. After these, it will remain only to show that the combinatorial bijection established in [14, §4] has further structure, and this will be shown in Section 5 .

An element $v$ of $I(d)$ remains fixed throughout this section.

Let $S^{v}$ denote the set of monomials in $\mathfrak{R}^{v}$ and $T^{v}$ the set of monomials in $\mathfrak{N}^{v}$. Let $\mathrm{SM}^{v, v}$ denote the set of $v$-compatible standard monomials that are anti-dominated by $v$ : a standard monomial $\theta_{1} \geq \ldots \geq \theta_{t}$ is anti-dominated by $v$ if $\mathfrak{b o t}\left(\theta_{t}\right) \geq v$.

\footnotetext{
${ }^{4}$ This is a consequence of Theorem 3.7 It is easy to see that the $p_{\mathfrak{w}}$ such that $w \geq$ top (w) vanish on $X_{w}$. Since all standard monomials form a basis for the homogeneous coordinate ring of $\mathfrak{M}_{d}(V) \subseteq \mathbb{P}\left(\wedge^{d} V\right)$, it follows that $w$-dominated standard monomials in admissible pairs span the quotient ring by the ideal generated by such $p_{\mathfrak{w}}$. Since such monomials are linearly independent in the homogeneous coordinate ring of $X_{w}$, the desired result follows.
} 
Define the domination map from $T^{v}$ to $I(d)$ by sending a monomial in $\mathfrak{N}^{v}$ to the least element that dominates it 5 Define the domination map from $S M^{v, v}$ to $I(d)$ by sending $\theta_{1} \geq \ldots \geq \theta_{t}$ to $\operatorname{top}\left(\theta_{1}\right)$. Both these maps take, by definition, the value $v$ on the empty monomial.

The desired bijection follows from the following proposition.

Proposition 4.1. There is a bijection between $S M^{v, v}$ and $T^{v}$ that respects domination and degree.

By the above proposition, we have, for $w$ in $I(d)$ with $w \geq v$, a bijection $\mathrm{SM}_{w}^{v, v}(m) \cong T_{w}^{v}(m)$, where $\mathrm{SM}_{w}^{v, v}(m)$ is the set of $w$-dominated elements of $\mathrm{SM}^{v, v}$ that have degree $m$, and similarly $T_{w}^{v}(m)$ is the set of $w$-dominated elements of $T^{v}$ that have degree $m$.

Now let $U^{v}$ denote the set of monomials in $\mathfrak{R}^{v} \backslash \mathfrak{N}^{v}$ (that is, in pairs $(r, c)$ of $\mathfrak{R}^{v}$ with $r<c$ ), and $S M_{v}^{v}$ the set of $v$-compatible and $v$-dominated standard monomials. As explained below, the "mirror image" of the bijection $S M^{v, v} \cong T^{v}$ gives a bijection $S M_{v}^{v}(m) \cong U^{v}(m)$, where $S M_{v}^{v}(m)$ and $U^{v}(m)$ denote respectively the sets of elements of degree $m$ of $S M_{v}^{v}$ and $U^{v}$.

Putting these bijections together, we get the desired bijection:

$$
\begin{aligned}
\mathrm{SM}_{w}^{v}(m) & =\bigcup_{j=0}^{m} S M_{w}^{v, v}(j) \times S M_{v}^{v}(m-j) \\
& \cong \bigcup_{j=0}^{m} T_{w}^{v}(j) \times U^{v}(m-j) \\
& =S_{w}^{v}(m) .
\end{aligned}
$$

Here the first equality is obtained by splitting a $v$-compatible standard monomial $\theta_{1} \geq \ldots \geq \theta_{t}$ into two parts $\theta_{1} \geq \ldots \geq \theta_{p}$ and $\theta_{p+1} \geq \ldots \geq \theta_{t}$, where $p$ is the largest integer, $1 \leq p \leq t$, with $\mathfrak{b} \mathfrak{o t}\left(\theta_{p}\right) \geq v$. The last equality is obtained by writing a monomial of degree $m$ in $\mathfrak{R}^{v}$ as a product of two monomials, one in $\mathfrak{N}^{v}$ and the other in $\mathfrak{R}^{v} \backslash \mathfrak{N}^{v}$, the sum of their degrees being $m$.

Let us now briefly explain how to take the "mirror image". Recall that $j^{*}:=$ $2 d-j+1$ for an integer $j, 1 \leq j \leq 2 d$. For $u=\left(u_{1}, \ldots, u_{d}\right)$ in $I(d)$, define the dual $u^{*}$ by $u^{*}:=\left(u_{d}^{*}, \ldots, u_{1}^{*}\right)$. This dual map on $I(d)$ is an order reversing involution. It induces a bijection $S M_{v}^{v} \cong S M^{v^{*}, v^{*}}$ by associating to $\theta_{1} \geq \ldots \geq \theta_{t}$ the element $\theta_{t}^{*} \geq \ldots \geq \theta_{1}^{*}$ - for an admissible pair $\theta=(\mathfrak{t o p}(\theta), \mathfrak{b o t}(\theta)), \theta^{*}$ denotes of course the admissible pair $\left(\mathfrak{b o t}(\theta)^{*}, \mathfrak{t o p}(\theta)^{*}\right)$. The sum of the $v$-degrees of $\theta_{1}, \ldots, \theta_{t}$ equals the sum of the $v^{*}$-degrees of $\theta_{t}^{*}, \ldots, \theta_{1}^{*}$, so that we get a bijection $S M_{v}^{v}(m) \cong$ $S M^{v^{*}, v^{*}}(m)$.

For an element $(r, c)$ in $\mathfrak{N}^{v^{*}}$, define the dual to be the element $(c, r)$. Since $v \in I(d)$, we have $v^{*}=[2 d] \backslash v$. Thus, it follows that $(c, r)$ belongs to $\mathfrak{R}^{v} \backslash \mathfrak{N}^{v}$. This induces a degree preserving bijection $T^{v^{*}} \cong U^{v}$. Putting this together with the bijection of the previous paragraph and the one given by Proposition 4.1 (for $v^{*}$ in place of $v$ ), we have

$$
S M_{v}^{v}(m) \cong S M^{v^{*}, v^{*}}(m) \cong T^{v^{*}}(m) \cong U^{v}(m) .
$$

\footnotetext{
${ }^{5}$ The poset $I(d)$ has a largest element, namely $(d+1, \ldots, 2 d)$, and this clearly dominates all monomials. The glb (greatest lower bound) in $I(d, 2 d)$ of a set of elements in $I(d)$ belongs to $I(d)$ since $\operatorname{glb}(u, x)^{\#}=\operatorname{glb}\left(u^{\#}, x^{\#}\right)$ for $u, x$ in $I(d, 2 d)$.
} 
The proof of Theorem 2.1 is thus reduced to the the proof of Proposition 4.1.

4.1. Proof of Proposition 4.1, The arguments in Section 3 and in the present section thus far have shown that Theorem 2.1 follows from Proposition 4.1. To prove the proposition, we will exploit the work already done in [14. More precisely, we will deduce the proposition from its earlier version stated in the paragraphs following Proposition 4.2 of that paper. As alluded to in the Introduction, the main ingredient in the deduction is showing that the bijection in the earlier version respects the involution induced on the combinatorial entities by the skew-symmetric form; see Lemma 4.5 and Proposition 5.5 below for the precise statements. We also need Lemma 5.15 which roughly speaking is a symmetry property of domination.

Remark 4.2. Proposition 4.1 and its earlier version are worded exactly alike. The difference lies in the meaning attached to the symbols. To distinguish the two meanings, we use in this section a tilde sign over these symbols to indicate that the symbol in question has the meaning given to it in [14. However, in Section 5 , the notation and terminology of that paper will throughout be in force, and so the tilde sign will be omitted.

Consider $v$ as an element of $I(d, 2 d)$. A standard monomial in $I(d, 2 d)$ is a totally ordered sequence $\theta_{1} \geq \ldots \geq \theta_{t}$ of elements of $I(d, 2 d)$. Such a monomial is $v$-compatible if each $\theta_{j}$ is comparable to $v$ but no $\theta_{j}$ equals $v$; it is anti-dominated by $v$ if $\theta_{t} \geq v$.

Notation 4.3. In keeping with Remark 4.2 , we have the following:

$\widetilde{\mathrm{SM}^{v, v}}$ denotes the set of $v$-compatible standard monomials in $I(d, 2 d)$ antidominated by $v$.

$\widetilde{\Re^{v}}$ denotes the set of ordered pairs $(r, c)$ such that $r \in[2 d] \backslash v$ and $c \in v$.

$\widetilde{\mathfrak{N}^{v}}$ denotes the subset of $\widetilde{\mathfrak{R}^{v}}$ consisting of those $(r, c)$ with $r>c$.

$\widetilde{T^{v}}$ denotes the set of monomials in $\widetilde{\mathfrak{N}^{v}}$.

Consider the natural injection $\mathrm{SM}^{v, v} \rightarrow \widetilde{\mathrm{SM}^{v, v}}$ that maps $\theta_{1} \geq \ldots \geq \theta_{t}$ to $\mathfrak{t o p}\left(\theta_{1}\right) \geq \mathfrak{b} \mathfrak{b o t}\left(\theta_{1}\right) \geq \ldots \geq \mathfrak{t o p}\left(\theta_{t}\right) \geq \mathfrak{b} \mathfrak{b o t}\left(\theta_{t}\right)$, except that $\mathfrak{b o t}\left(\theta_{t}\right)$ is omitted if it equals $v$. This map preserves domination and doubles degree. Composing this with the domination and degree preserving bijection $\widetilde{\mathrm{SM}^{v, v}} \rightarrow \widetilde{T^{v}}$ of [14, §4], we get an injection of $\mathrm{SM}^{v, v}$ into $\widetilde{T^{v}}$.

To describe the image of $\mathrm{SM}^{v, v}$ in $\widetilde{T^{v}}$, we introduce a definition. For $\alpha=(r, c)$ in $\widetilde{\mathfrak{R}^{v}}$, set $\alpha^{\#}:=\left(c^{*}, r^{*}\right)$. The map $\alpha \mapsto \alpha^{\#}$ is an involution on $\widetilde{\mathfrak{R}^{v}}$ and on $\widetilde{\mathfrak{N}^{v}}$. Elements of the form $\left(r, r^{*}\right)$ of $\widetilde{\mathfrak{N}^{v}}$ are referred to as belonging to the "diagonal", and the set of diagonal elements of $\widetilde{\mathfrak{N}^{v}}$ is denoted $\mathfrak{d}^{v}$.

Definition 4.4. A monomial $\mathfrak{S}$ of $\widetilde{T^{v}}$ is special if

(1) $\mathfrak{S}=\mathfrak{S}^{\#}$ and

(2) the multiplicity of any diagonal element in $\mathfrak{S}$ is even.

Equivalently, $\mathfrak{S}$ is special if there exists $\mathfrak{T}$ in $\widetilde{T^{v}}$ with $\mathfrak{S}=\mathfrak{T} \cup \mathfrak{T} \#$. The set of special monomials is denoted $\mathfrak{E}$.

The following is the main technical result of the present paper. It is an immediate corollary of Proposition 5.5 below. Here we use it to prove Proposition 4.1 .

Lemma 4.5. The image of $\mathrm{SM}^{v, v}$ in $\widetilde{T^{v}}$ is the set $\mathfrak{E}$ of special monomials. 
In other words, there is a domination preserving and degree doubling bijective map from $\mathrm{SM}^{v, v}$ to $\mathfrak{E}$.

On the other hand, there is a domination preserving and degree halving bijective map from $\mathfrak{E}$ to $T^{v}$. Given $\mathfrak{S}$ in $\mathfrak{E}$, to get the corresponding element of $T^{v}$, replace those $(r, c)$ of $\mathfrak{S}$ with $r>c^{*}$ by $\left(c^{*}, r^{*}\right)$ and then take the (positive) square root. This clearly halves the degrees. The map in the other direction is obvious: given a monomial of $T^{v}$, replace each $(r, c)$ by $(r, c)\left(c^{*}, r^{*}\right)$-in other words, a monomial $\mathfrak{P}$ of $T^{v}$ is mapped to $\mathfrak{P} \cup \mathfrak{P}^{\#}$.

To see that this bijection from $\mathfrak{E}$ to $T^{v}$ preserves domination, let $\mathfrak{T}$ be a special monomial and $\mathfrak{P}$ the corresponding monomial in $T^{v}$. Let $y$ and $z$ be the images respectively of $\mathfrak{T}$ and $\mathfrak{P}$ under the domination maps. Then $y$ is the first coordinate of $\pi(\mathfrak{T})$, where $\pi$ is the map defined in [14, §4]; see Proposition 4.1 (4) of [14]. Since $\mathfrak{T}=\mathfrak{T}^{\#}$, it follows from Proposition 5.6 below that $y=y^{\#}$. This is equivalent to saying that $y$ belongs to $I(d)$.

That $y$ dominates $\mathfrak{P}$ is clear: any $v$-chain in $\mathfrak{P}$ is also a $v$-chain in $\mathfrak{T}$. Since $y$ is in $I(d)$, it follows from the definition of the domination map on $T^{v}$ that $y \geq z$. That $z \geq y$ follows from Lemma 5.15 below: $z^{\#}=z, \mathfrak{P}=\mathfrak{P}^{\text {up }}$, and $z$ dominates $\mathfrak{P}$, so that $z$ dominates $\mathfrak{P} \cup \mathfrak{P}^{\#}=\mathfrak{T}$.

Composing the two bijective maps $\mathrm{SM}^{v, v} \rightarrow \mathfrak{E}$ and $\mathfrak{E} \rightarrow T^{v}$ above gives us a domination and degree preserving bijection $\mathrm{SM}^{v, v} \rightarrow T^{v}$. Proposition 4.1 is thus proved.

\section{Completion of the ProOF}

The purpose of this section is to establish the statements used in the proof of Proposition 4.1. The terminology and notation of [14] will be in force throughout.

An element $v$ of $I(d, n)$ remains fixed throughout. The symbol $w$ will denote an arbitrary element of $I(d, n)$ satisfying $w \geq v, \mathfrak{S}$ an arbitrary monomial in $\mathfrak{N}^{v}$, and $\mathfrak{S}_{w}$ the unique subset of $\mathfrak{N}^{v}$ defined by Proposition 5.1 below.

Let $(r, c)$ and $(R, C)$ be elements of $\mathfrak{N}^{v}$. They are comparable if either they are equal or $(R, C)>(r, c)$ (which means that $R>r$ and $C<c)$ or $(r, c)>(R, C)$. We say that $(R, C)$ dominates $(r, c)$ if $R \geq r$ and $C \leq c$.

\subsection{Recall.}

Proposition 5.1. To each element $w$ of $I(d, n)$ satisfying $w \geq v$ there is associated a unique subset $\mathfrak{S}_{w}=\left\{\left(r_{1}, c_{1}\right), \ldots,\left(r_{p}, c_{p}\right)\right\}$ of $\mathfrak{N}^{v}$ satisfying conditions $\mathrm{A}-\mathrm{C}$ below:
A. $r_{i} \neq r_{j}$ and $c_{i} \neq c_{j}$ for $i \neq j$.
B. If $r_{i}<r_{j}$, then either $c_{j}<c_{i}$ or $r_{i}<c_{j}$.
C. $w=\left(v \backslash\left\{c_{1}, \ldots, c_{p}\right\}\right) \cup\left\{r_{1}, \ldots, r_{p}\right\}$.

Furthermore, $\mathfrak{S}_{w}$ also satisfies conditions $\mathrm{D}$ and $\mathrm{E}$ below:

D. The $v$-degree of $w$ equals the cardinality $p$ of $\mathfrak{S}_{w}$.

E. $w$ is the smallest element of $I(d, n)$ to dominate $\mathfrak{S}_{w}$.

Proof. The existence and uniqueness of $\mathfrak{S}_{w}$ is the substance of Proposition 4.3 of 14. That D holds is obvious. For E, see Lemma 5.5 of [14].

Remark 5.2. If $\mathfrak{S}$ is a subset of $\mathfrak{N}^{v}$ that satisfies condition A of Proposition 5.1, the equation in item $\mathrm{C}$ can be taken to be the definition of an element $w$ of $I(d, n)$. If $\mathfrak{S}$ satisfies $B$ also, then $\mathfrak{S}=\mathfrak{S}_{w}$ for this $w$. Let $x$ be an element of $I(d, n)$ and 
$\mathfrak{S}$ a subset of $\mathfrak{S}_{x}$. Then $\mathfrak{S}$ evidently satisfies A and B. Therefore $\mathfrak{S}=\mathfrak{S}_{w}$ for some $w$. It follows from $\mathrm{E}$ that $x \geq w$.

Lemma 5.3. Let $\mathfrak{B}$ and $\mathfrak{C}$ be distinct blocks of a monomial $\mathfrak{S}$. Then $\mathfrak{B} \cup \mathfrak{B}^{\prime}$ and $\mathfrak{C} \cup \mathfrak{C}^{\prime}$ are disjoint.

Proof. For $\mathfrak{B}$ and $\mathfrak{C}$ both belonging to the same partition $\mathfrak{S}_{j}$ of $\mathfrak{S}$, this follows from the proof of Lemma 4.10 of [14]; see the last line of that proof. Now suppose that $\mathfrak{B} \subseteq \mathfrak{S}_{i}$ and $\mathfrak{C} \subseteq \mathfrak{S}_{j}$, and that $(r, c)$ belongs to both $\mathfrak{B} \cup \mathfrak{B}^{\prime}$ and $\mathfrak{C} \cup \mathfrak{C}^{\prime}$. Assume without loss of generality that $i<j$. From the definition of $\mathfrak{C}^{\prime}$, it follows that there exists an element $\left(r^{\prime}, c\right)$ in $\mathfrak{C}$ with $r^{\prime} \geq r$. From the definition of the partitions $\mathfrak{S}_{k}$, it follows that there exists $(a, b)$ in $\mathfrak{S}_{i}$ such that $(a, b)>\left(r^{\prime}, c\right)$. Now $(a, b)>(r, c)$ and both of them belong to $\mathfrak{S}_{i} \cup \mathfrak{S}_{i}^{\prime}$, a contradiction to Lemma 4.10 of [14].

Corollary 5.4. Given $(r, c)$ in $\mathfrak{S} \cup \mathfrak{S}^{\prime}$, there exists a unique block $\mathfrak{B}$ of $\mathfrak{S}$ such that $(r, c)$ belongs to $\mathfrak{B} \cup \mathfrak{B}^{\prime}$.

5.2. Statement of the main proposition. From now on we will assume that $n=2 d$ and that $v=v^{\#}$ (or equivalently, that $v$ belongs to $I(d)$ ). The map $\pi$ of [14, §4.2] is defined only on nonempty monomials. We extend the definition by setting $\pi(\mathfrak{S})=(v, \mathfrak{S})$ if $\mathfrak{S}$ is empty. Similarly we extend the definition of the map $\phi$ of [14, $\S 4.4]$ by setting $\phi(v, \mathfrak{T})=\mathfrak{T}$ if $\mathfrak{T}$ is empty.

The following proposition has as an immediate corollary Lemma 4.5 which was the main ingredient in the proof of Proposition 4.1. Its proof will be given in Subsections 5.4 and 5.5 after some preliminaries in Subsection 5.3

Proposition 5.5. Let $\mathfrak{S}$ be a special monomial. Set

$$
\pi(\mathfrak{S})=\left(t, \mathfrak{S}^{\prime}\right) \quad \text { and } \quad \pi\left(\mathfrak{S}^{\prime}\right)=(u, \mathfrak{T}) .
$$

Then

(1) $t \geq u \geq v, t^{\#}=t, u^{\#}=u$, and the $(1, \ldots, d)$-degrees of $t$ and $u$ are equal.

(2) $\mathfrak{T}$ is special and $u$ dominates $\mathfrak{T}$.

Conversely, if $t$ and $u$ are elements of $I(d, 2 d)$ and $\mathfrak{T}$ a monomial such that the two conditions above are satisfied, then $\phi(t, \phi(u, \mathfrak{T}))$ is special.

5.3. Behavior under the operation \#. In this subsection we investigate the behavior of the combinatorial constructions of [14, $\S 4$ ] under the operation \#.

If $\beta$ is $j$-deep in $\mathfrak{S}$, then $\beta^{\#}$ is $j$-deep in $\mathfrak{S}^{\#}$. Thus the partitions $\mathfrak{S}_{j}$ of $\mathfrak{S}$ are respected by the hash operation: $\left(\mathfrak{S}_{j}\right)^{\#}=\left(\mathfrak{S}^{\#}\right)_{j}$. It is also easy, given the definitions, to verify the following:

- If $\mathfrak{B}$ is a block of $\mathfrak{S}_{j}$, then $\mathfrak{B}^{\#}$ is a block of $\mathfrak{S}_{j}^{\#}$.

- $w\left(\mathfrak{B}^{\#}\right)=w(\mathfrak{B})^{\#}$.

- $\left(\mathfrak{B}^{\#}\right)^{\prime}=\left(\mathfrak{B}^{\prime}\right)^{\#}$.

These observations amount to a proof of the following proposition.

Proposition 5.6. The map $\pi$ respects \#. More precisely, if $\pi(\mathfrak{S})=\left(w, \mathfrak{S}^{\prime}\right)$, then $\pi\left(\mathfrak{S}^{\#}\right)=\left(w^{\#}, \mathfrak{S}^{\prime \#}\right)$.

Let us now show that the map $\phi$ also respects \#. For this we need

Proposition 5.7. The association $w \mapsto \mathfrak{S}_{w}$ respects \#, that is, $\left(\mathfrak{S}_{w}\right)^{\#}=\mathfrak{S}_{w^{\#}}$. In particular, $w^{\#}=w$ if and only if $\left(\mathfrak{S}_{w}\right)^{\#}=\mathfrak{S}_{w}$. 
Proof. The result follows from Proposition [5.1 $\left(\mathfrak{S}_{w}\right)^{\#}$ satisfies conditions A, B and $\mathrm{C}$ of that proposition with $w$ replaced by $w^{\#}$ in condition $\mathrm{C}$.

Let $\mathfrak{T}$ be a monomial in $\mathfrak{N}^{v}$ and $w$ an element of $I(d, 2 d)$ such that $w \geq v$ and $w$ dominates $\mathfrak{T}$. The following are easily verified from the definitions:

- $\left(\mathfrak{S}_{w}^{j}\right)^{\#}=\mathfrak{S}_{w^{\#}}^{j}$ and so $\left(w^{j}\right)^{\#}=\left(w^{\#}\right)^{j}$.

- $\left(\mathfrak{T}_{j}^{w}\right)^{\#}=\left(\mathfrak{T}^{\#}\right)_{j}^{w^{\#}}$.

- $\left(\mathfrak{P}_{\beta}\right)^{\#}$ is the piece of $\mathfrak{T}^{\#}$ corresponding to $\beta^{\#}$, for $\beta$ an element of $\mathfrak{S}_{w}$.

- $\left(\left(\mathfrak{T}_{j}^{w}\right)^{\star}\right)^{\#}=\left(\left(\mathfrak{T}^{\#}\right)_{j}^{w^{\#}}\right)^{\star}$.

These observations amount to a proof of the following proposition.

Proposition 5.8. The map $\phi$ respects \#: more precisely, if $\phi(w, \mathfrak{T})=\mathfrak{S}$, then $\phi\left(w^{\#}, \mathfrak{T}^{\#}\right)=\mathfrak{S}^{\#}$.

Proposition 5.9. Suppose that $w=w^{\#}$ (or equivalently, by Proposition 5.7, $\mathfrak{S}_{w}=$ $\left.\mathfrak{S}_{w}^{\#}\right)$. Then for an element $(r, c)$ of $\mathfrak{S}_{w}$, either (i) $c<r \leq d$, or (ii) $d<c<r$, or (iii) $r^{\star}=c \leq d<r$. In particular, the $\epsilon$-degree of $w$ equals the number of elements of $\mathfrak{S}_{w}$ belonging to the diagonal.

Proof. Suppose that $c \leq d$ and $r>d$. Since $\mathfrak{S}_{w}=\mathfrak{S}_{w}^{\#},\left(c^{*}, r^{*}\right)$ belongs to $\mathfrak{S}_{w}$. If $r<c^{*}$, then $r^{*}>c$, and the presence of both $(r, c)$ and $\left(c^{*}, r^{*}\right)$ in $\mathfrak{S}_{w}$ is a violation of condition $\mathrm{E}$ of Proposition [5.1, a contradiction. If $c^{*}<r$, then $c>r^{*}$, and again there is a similar contradiction. Thus $c=r^{*}$.

Proposition 5.10. Let $\mathfrak{B}$ be a block of a monomial $\mathfrak{S}$ satisfying $\mathfrak{S}=\mathfrak{S}^{\#}$.

(A) The following are equivalent:

(1) $\mathfrak{B}=\mathfrak{B}^{\#}$.

(2) $w(\mathfrak{B})$ lies in the diagonal.

(3) $\mathfrak{B} \cup \mathfrak{B}^{\prime}$ meets the diagonal.

(B) Suppose that the conditions in (A) above are met. Then there is a unique element of the diagonal in $\mathfrak{B} \cup \mathfrak{B}^{\prime}$, and the multiplicities of that element in $\mathfrak{B}$ and $\mathfrak{B}^{\prime}$ differ by 1 .

Proof. (A): As noted in the proof of Proposition [5.6. $\mathfrak{B}^{\#}$ is a block of $\mathfrak{S}^{\#}=\mathfrak{S}$, $w\left(\mathfrak{B}^{\#}\right)=w(\mathfrak{B})^{\#}$, and $\left(\mathfrak{B}^{\#}\right)^{\prime}=\left(\mathfrak{B}^{\prime}\right)^{\#}$.

$(1) \Rightarrow(2): w(\mathfrak{B})^{\#}=w(\mathfrak{B} \#)=w(\mathfrak{B})$, which means that $w(\mathfrak{B})$ is on the diagonal.

$(2) \Rightarrow(1)$ : We have $w\left(\mathfrak{B}^{\#}\right)=w(\mathfrak{B})^{\#}=w(\mathfrak{B})$. If $\mathfrak{B}$ and $\mathfrak{B}^{\#}$ are distinct blocks, then $w(\mathfrak{B})$ and $w\left(\mathfrak{B}^{\#}\right)$ cannot share a row or column indexCorollary 4.13 of [14] - let alone be equal.

$(3) \Rightarrow(1)$ : It follows from the hypothesis that $\mathfrak{B} \cup \mathfrak{B}^{\prime}$ and $\mathfrak{B}^{\#} \cup\left(\mathfrak{B}^{\#}\right)^{\prime}$ meet. By Corollary [5.4, $\mathfrak{B}=\mathfrak{B}^{\#}$.

$(1) \Rightarrow(3)$ : Since $\mathfrak{B}=\mathfrak{B}^{\#}$, there is an element $(r, s)$ of $\mathfrak{B}$ with $r \leq s^{*}$. Let $r$ be the maximal row index of such an element of $\mathfrak{B}$. A portion of the arrangement of the elements of $\mathfrak{B}$ in ascending order of row and column indices looks like this:

$$
\ldots,(a, b), \underbrace{\left(r, r^{\star}\right), \ldots,\left(r, r^{\star}\right)}_{m \text { times }},\left(b^{\star}, a^{\star}\right), \ldots
$$


where either $a<r$ or $b<r^{\star}$. If $m>0$, then $\mathfrak{B}$ meets the diagonal and we are done. If $m=0$, then $a=r$, and the portion of $\mathfrak{B}^{\prime}$ corresponding to the one above of $\mathfrak{B}$ looks like this:

$$
\ldots,(?, b),\left(a, a^{\star}\right),\left(b^{\star}, ?\right), \ldots
$$

so that $\left(a, a^{\star}\right)=\left(r, r^{\star}\right)$ belongs to $\mathfrak{B}^{\prime}$.

(B): Since elements of the diagonal are comparable but no two distinct elements of $\mathfrak{B} \cup \mathfrak{B}^{\prime}$ are (the latter statement is immediate from the definitions; or see Lemma 4.10 of [14]), there is at most one diagonal element in $\mathfrak{B} \cup \mathfrak{B}^{\prime}$. Since there is at least one such element by hypothesis, there is a unique such element.

Let $\left(r, r^{\star}\right)$ be the unique diagonal element $\mathfrak{B} \cup \mathfrak{B}^{\prime}$. Proceed as in the proof of $(1) \Rightarrow(3)$ above. If $m=0$, then $\left(a, a^{*}\right)=\left(r, r^{*}\right)$ belongs to $\mathfrak{B}^{\prime}$. Further $b<r^{*}$ and $b^{*}>r$, so that the multiplicity of $\left(r, r^{\star}\right)$ in $\mathfrak{B}^{\prime}$ is 1 and we are done.

If $m>0$, the portion of $\mathfrak{B}^{\prime}$ corresponding to the one above of $\mathfrak{B}$ looks like this:

$$
\ldots,(?, b),\left(a, r^{\star}\right), \underbrace{\left(r, r^{\star}\right), \ldots,\left(r, r^{\star}\right)}_{m-1 \text { times }},\left(r, a^{\star}\right),\left(b^{\star}, ?\right), \ldots
$$

The multiplicity of $\left(r, r^{\star}\right)$ in $\mathfrak{B}^{\prime}$ is therefore either $m+1$ or $m-1$ depending upon whether or not $a$ equals $r$.

Corollary 5.11. Let $\mathfrak{S}=\mathfrak{S}^{\#}$ and $\left(r, r^{\star}\right)$ be an element of the diagonal belonging to $\mathfrak{S} \cup \mathfrak{S}^{\prime}$. Then the multiplicities of $\left(r, r^{\star}\right)$ in $\mathfrak{S}$ and $\mathfrak{S}^{\prime}$ differ by 1 .

Proof. By Corollary 5.4, there is a unique block $\mathfrak{B}$ of $\mathfrak{S}$ such that $\left(r, r^{\star}\right)$ belongs to $\mathfrak{B} \cup \mathfrak{B}^{\prime}$. The multiplicities of $\left(r, r^{\star}\right)$ in $\mathfrak{S}$ and $\mathfrak{S}^{\prime}$ equal respectively those in $\mathfrak{B}$ and $\mathfrak{B}^{\prime}$, and these differ by 1 by the proposition above.

Definition 5.12. For $\alpha=(r, c)$ in $\mathfrak{N}^{v}$, set

$$
\alpha^{\text {up }}:=\left\{\begin{array}{cl}
\alpha & \text { if } r \leq c^{\star}, \\
\alpha^{\#}=\left(c^{\star}, r^{\star}\right) & \text { if } r>c^{*},
\end{array} \quad \alpha^{\text {down }}:=\left\{\begin{array}{cc}
\alpha & \text { if } r \geq c^{\star}, \\
\alpha^{\#}=\left(c^{\star}, r^{\star}\right) & \text { if } r<c^{*} .
\end{array}\right.\right.
$$

Proposition 5.13. Let $\alpha$ be an element of $\mathfrak{N}^{v}$ and $w$ an element of $I(d, 2 d)$ with $w \geq v$. If both $\alpha^{\text {up }}$ and $\alpha^{\text {down }}$ belong to $\mathfrak{S}_{w}$ and both of them dominate an element $\beta$ of $\mathfrak{N}^{v}$, then $\alpha^{\text {up }}=\alpha^{\text {down }}$. In particular, if $w=w^{\#}$ and $\alpha$ in $\mathfrak{S}_{w}$ dominates an element on the diagonal, then $\alpha$ belongs to the diagonal.

Proof. Write $\alpha^{\text {up }}=(R, C)$ and $\beta=(r, c)$. Then $C \leq R^{*} \leq c<r \leq R \leq C^{*}$. Here $C \leq R^{*}$ and $R \leq C^{*}$ because $\alpha^{\text {up }}=(R, C), R^{*} \leq c$ because $\alpha^{\text {down }}=\left(C^{*}, R^{*}\right)$ dominates $\beta, c<r$ because $\beta$ belongs to $\mathfrak{N}^{v}$, and $r \leq R$ because $\alpha^{\text {up }}$ dominates $\beta$. Unless $R=C^{*}$ the presence of both $\alpha^{\text {up }}$ and $\alpha^{\text {down }}$ in $\mathfrak{S}_{w}$ leads to a violation of condition B of Proposition 5.1 by $\mathfrak{S}_{w}$, a contradiction.

Corollary 5.14. If $\alpha_{1}>\ldots>\alpha_{p}$ is the $v$-chain of diagonal elements in $\mathfrak{S}_{w}$ for an element $w=w^{\#}$, then $\alpha_{j}$ is $j$-deep but not $j+1$-deep in $\mathfrak{S}_{w}$.

Lemma 5.15. If $w^{\#}=w$ and $w$ dominates a monomial $\mathfrak{S}$ with $\mathfrak{S}=\mathfrak{S}^{\text {up }}$ (that is, $\alpha=\alpha^{u p}$ for every $\left.\alpha \in \mathfrak{S}\right)$, then $w$ also dominates $\mathfrak{S} \cup \mathfrak{S} \#$.

Proof. Let $\beta_{1}>\ldots>\beta_{p}$ be a $v$-chain in $\mathfrak{S} \cup \mathfrak{S} \#$. We need to show that this chain is dominated by a $v$-chain in $\mathfrak{S}_{w}$. We may assume without loss of generality that $\beta_{p}^{\text {up }}=\beta_{p}$. If $\beta_{j}^{\text {up }}=\beta_{j}$ for all $j$, then the desired result follows immediately from the hypothesis. Otherwise, let $k \in[p]$ be the largest such that $\beta_{k}^{\text {down }}=\beta_{k}$. 
Choose a $v$-chain $\alpha_{1}>\ldots>\alpha_{p}$ in $\mathfrak{S}_{w}$ such that it dominates $\beta_{1}^{\text {up }}>\ldots>\beta_{p}^{\text {up }}$. Replacing $\alpha_{1}>\ldots>\alpha_{p}$ by $\alpha_{1}^{\text {up }}>\ldots>\alpha_{p}^{\text {up }}$, we may assume that $\alpha_{j}^{\text {up }}=\alpha_{j}$. It follows from Proposition 5.13 that $\alpha_{j}$ lives on the diagonal for $1 \leq j \leq k$ : $\alpha_{k}^{\text {down }}>\beta_{k}^{\text {down }}=\beta_{k}>\beta_{k+1}$ and $\alpha_{k}^{\text {up }}>\beta_{k}^{\text {up }}>\beta_{k+1}^{\text {up }}=\beta_{k+1}$ imply that $\alpha_{k}$ belongs to the diagonal, $\alpha_{k-1}>\alpha_{k}$ implies that $\alpha_{k-1}$ belongs to the diagonal, etc. Thus $\alpha_{j}$ dominates $\beta_{j}$ for all $j \in[p]$.

5.4. Proof of the first half of Proposition 5.5. By Proposition 5.6 above, $\pi$ respects the \# operation. Hence it follows that $t^{\#}=t,\left(\mathfrak{S}^{\prime}\right)^{\#}=\mathfrak{S}^{\prime}, u^{\#}=u$ and $(\mathfrak{T})^{\#}=\mathfrak{T}$. Also, it follows from 1, 3 and 4 of Proposition 4.1 of [14] that $t \geq u \geq v$, and that $u$ dominates $\mathfrak{T}$. Thus it remains only to prove the following:

(a) $\mathfrak{S}_{t}$ meets the diagonal in as many points as $\mathfrak{S}_{u}$. This will imply, by Proposition 5.9. that the $\epsilon$-degrees of $t$ and $u$ are equal and hence that $(t, u)$ is an admissible pair.

(b) $\mathfrak{T}$ is special.

We first prove (a). If $\left(r, r^{\star}\right)$ and $\left(s, s^{\star}\right)$ are elements of the diagonal $\mathfrak{d}^{v}$, then either $\left(r, r^{\star}\right)>\left(s, s^{\star}\right)$ or the other way around. The elements of $\mathfrak{S}_{u} \cap \mathfrak{d}^{v}$ therefore form a $v$-chain. Let $\beta_{1}>\ldots>\beta_{p}$ be all the elements of $\mathfrak{S}_{u} \cap \mathfrak{d}^{v}$. By Proposition 5.1 (E), $t$ dominates $\mathfrak{S}_{u}$. By Lemma 4.5 of [14], there exists a $v$-chain $\alpha_{1}>\ldots>\alpha_{p}$ in $\mathfrak{S}_{t}$ that dominates $\beta_{1}>\ldots>\beta_{p}$. By Proposition 5.13, the $\alpha_{j}$ belong to $\mathfrak{d}^{v}$.

To complete the proof of (a), it remains to be seen that the cardinality of $\mathfrak{S}_{u} \cap \mathfrak{d}^{v}$ is not less than that of $\mathfrak{S}_{t} \cap \mathfrak{d}^{v}$. We will show in fact that $\mathfrak{S}^{\prime} \cap \mathfrak{d}^{v}$ has at least as many distinct elements as the cardinality of $\mathfrak{S}_{t} \cap \mathfrak{d}^{v}$. The blocks containing these elements will then clearly be distinct: elements of the diagonal are comparable, but no two distinct elements of a block are. Each such block $\mathfrak{C}$ satisfies $\mathfrak{C}^{\#}=\mathfrak{C}$, for blocks are disjoint and $\mathfrak{C}$ meets the diagonal. If $\mathfrak{C}$ and $\mathfrak{C}_{1}$ are distinct blocks, then $w(\mathfrak{C})$ and $w\left(\mathfrak{C}_{1}\right)$ are distinct (Corollary 4.13 of [14]) so there are distinct contributions to $\mathfrak{S}_{u}$ from these blocks. Finally, these contributions all lie on the diagonal by Proposition 5.10 .

Let $\beta$ be an element of $\mathfrak{S}_{t} \cap \mathfrak{d}^{v}$. Let $\mathfrak{B}$ be the block of $\mathfrak{S}$ with $w(\mathfrak{B})=\beta$. By Proposition 5.10, $\mathfrak{B} \cup \mathfrak{B}^{\prime}$ meets $\mathfrak{d}^{v}$. If $\mathfrak{B}$ meets $\mathfrak{d}^{v}$ at say $\beta^{\prime}$, then, since $\mathfrak{S}$ satisfies condition 2 of Definition 4.4, it follows from the definition of $\mathfrak{B}^{\prime}$ that $\mathfrak{B}^{\prime}$ also contains $\beta^{\prime}$. Thus we get an element $\beta^{\prime}$ in $\mathfrak{S}^{\prime} \cap \mathfrak{d}^{v}$ for every $\beta$ in $\mathfrak{S}_{t} \cap \mathfrak{d}^{v}$. The association $\beta \mapsto \beta^{\prime}$ is injective, for the association $\beta \mapsto \mathfrak{B}$ is injective (see the reason given in the last paragraph) and so is $\mathfrak{B} \mapsto \beta^{\prime}$ by Lemma 5.3. This finishes the proof of (a).

For use in the proof of (b), let us record a corollary of the proof of (a).

Corollary 5.16. A block $\mathfrak{B}$ of $\mathfrak{S}^{\prime}$ meets the diagonal if and only if $\mathfrak{B}=\mathfrak{B} \#$.

Proof. If $\mathfrak{B}$ meets the diagonal, then $\mathfrak{B}^{\#}=\mathfrak{B}$. The number of $\mathfrak{B}$ such that $\mathfrak{B}^{\#}=\mathfrak{B}$ is the cardinality of $\mathfrak{S}_{u} \cap \mathfrak{d}^{v}$. The number of $\mathfrak{B}$ that meet the diagonal is the cardinality of $\mathfrak{S}^{\prime} \cap \mathfrak{d}^{v}$. But we have seen that the cardinalities of $\mathfrak{S}_{u} \cap \mathfrak{d}^{v}$, $\mathfrak{S}^{\prime} \cap \mathfrak{d}^{v}$, and $\mathfrak{S}_{t} \cap \mathfrak{d}^{v}$ are equal.

We now turn to the proof of (b). Suppose that $\left(r, r^{\star}\right)$ belongs to $\mathfrak{T}$. Let $\mathfrak{B}$ be the block of $\mathfrak{S}^{\prime}$ such that $\left(r, r^{\star}\right)$ belongs to $\mathfrak{B}^{\prime}$. By Proposition 5.10 and the corollary above, $\mathfrak{B}$ meets the diagonal. Since elements of $\mathfrak{B} \cup \mathfrak{B}^{\prime}$ are incomparable (see Lemma 4.10 of [14]), it follows that $\left(r, r^{\star}\right)$ belongs to $\mathfrak{B}$ and so to $\mathfrak{S}^{\prime}$. By Corollary 5.11, the multiplicities of $\left(r, r^{\star}\right)$ in $\mathfrak{S}$ and $\mathfrak{S}^{\prime \prime}=\mathfrak{T}$ differ by 2,0 or -2 . 
5.5. Proof of the converse in Proposition 5.5. Set $\mathfrak{M}=\phi(u, \mathfrak{T})$ and $\mathfrak{N}=$ $\phi(t, \mathfrak{M})$. Since $\phi$ respects \# (see Proposition [5.8), it follows that $\mathfrak{M}^{\#}=\mathfrak{M}$ and $\mathfrak{N}^{\#}=\mathfrak{N}$. The only thing that takes some work is to show that $\mathfrak{N}$ satisfies condition (2) of Definition 4.4.

By Proposition 5.10, each diagonal element of $\mathfrak{N}$ belongs to a block $\mathfrak{B}$ of $\mathfrak{N}$ with $w(\mathfrak{B})$ on the diagonal. It therefore suffices to show that the multiplicity of a diagonal element in any such block of $\mathfrak{N}$ is even (possibly 0 ).

Let $\alpha_{1}>\ldots>\alpha_{p}$ and $\beta_{1}>\ldots>\beta_{p}$ be respectively the elements $\mathfrak{S}_{t} \cap \mathfrak{d}^{v}$ and $\mathfrak{S}_{u} \cap \mathfrak{d}^{v}$. Let $\mathfrak{P}_{1}, \ldots, \mathfrak{P}_{p}$ be the pieces of $\mathfrak{T}$ with respect to $u$ corresponding respectively to $\beta_{1}, \ldots, \beta_{p}$. Then $\mathfrak{P}_{j}^{\star}=\mathfrak{B}$ is a block of $\mathfrak{M}$ with $w\left(\mathfrak{P}_{j}^{\star}\right)=\mathfrak{B}_{j}$ and $\mathfrak{P}_{j}=\mathfrak{B}^{\prime}$; see the claim at the beginning of $\S 4.5$ of $[14$.

Since $\mathfrak{T}$ is special, it follows from Proposition 5.10 that $\mathfrak{P}_{j}^{\star}$ meets the diagonal with (positive) odd multiplicity. Denoting the diagonal element of $\mathfrak{P}_{j}^{\star}$ by $\delta_{j}$, we have $\delta_{1}>\ldots>\delta_{p}$; see Lemma 4.21 of [14]. By Proposition [5.13, the piece of $\mathfrak{M}$ with respect to $t$ to which $\delta_{j}$ belongs can only correspond to one of the $\alpha_{i}$.

No two distinct elements of a piece are comparable, so two different $\delta$ cannot belong to the piece corresponding to the same $\alpha$. Further, it follows from the definition of the way we break a monomial into pieces that if $i<j$ and $\delta_{i}, \delta_{j}$ belong respectively to the pieces corresponding to $\alpha_{k}$ and $\alpha_{l}$, then $k<l$. Thus $\delta_{j}$ belongs to the piece corresponding to $\alpha_{j}$.

Let $\mathfrak{B}_{1}, \ldots, \mathfrak{B}_{p}$ be the blocks of $\mathfrak{N}$ with $w\left(\mathfrak{B}_{j}\right)=\alpha_{j}$. Then $\mathfrak{B}_{j}^{\prime}$ is the piece of $\mathfrak{M}$ with respect to $t$ corresponding to $\alpha_{j}$; see the claim at the beginning of [14, $\S 4.5]$. By Proposition 5.10, the multiplicity of the diagonal element in $\mathfrak{B}_{j}$ is even (possibly 0).

\section{INTERPRETATIONS}

Fix elements $v, w$ in $I(d)$ with $v \leq w$. It follows from Corollary 2.2 that the multiplicity of the Schubert variety $X_{w}$ in $\mathfrak{M}_{d}(V)$ at the point $e^{v}$ can be interpreted as the cardinality of a certain set of nonintersecting lattice paths. We illustrate this by means of two examples. The justification for the interpretation is the same word-for-word as for the usual Grassmannian [14, §5] and so will be omitted. The interpretation leads immediately to a formula for the multiplicity involving binomial determinants. Specializing to the case $v=\epsilon=(1, \ldots, d)$, we recover Conca's result [4, Theorem 3.6].

Example 6.1. Let $d=23$. Consider

$$
v=(1,2,3,4,5,11,12,13,14,19,20,22,23,26,29,30,31,32,37,38,39,40,41)
$$

and

$$
w=(4,5,9,10,14,17,18,21,23,25,27,28,31,32,34,35,36,39,40,41,44,45,46)
$$

so that

$$
\begin{aligned}
\mathfrak{S}_{w}= & \{(9,3),(10,2),(17,13),(18,12),(21,20),(25,22), \\
& (27,26),(28,19),(34,30),(35,29),(36,11),(44,38),(45,37),(46,1)\} .
\end{aligned}
$$

We think of $v$ and $w$ as elements of $I(d, 2 d)$ and switch to the notation of [14 as we have done in Section 5. The grid depicting the points of $\mathfrak{N}^{v}$ is shown in Figure 1 The solid dots represent the points of $\mathfrak{S}_{w}$, this being the monomial associated to $w$ by Proposition 5.1. From each point $\beta$ of $\mathfrak{S}_{w}$ we draw a vertical line and a horizontal 


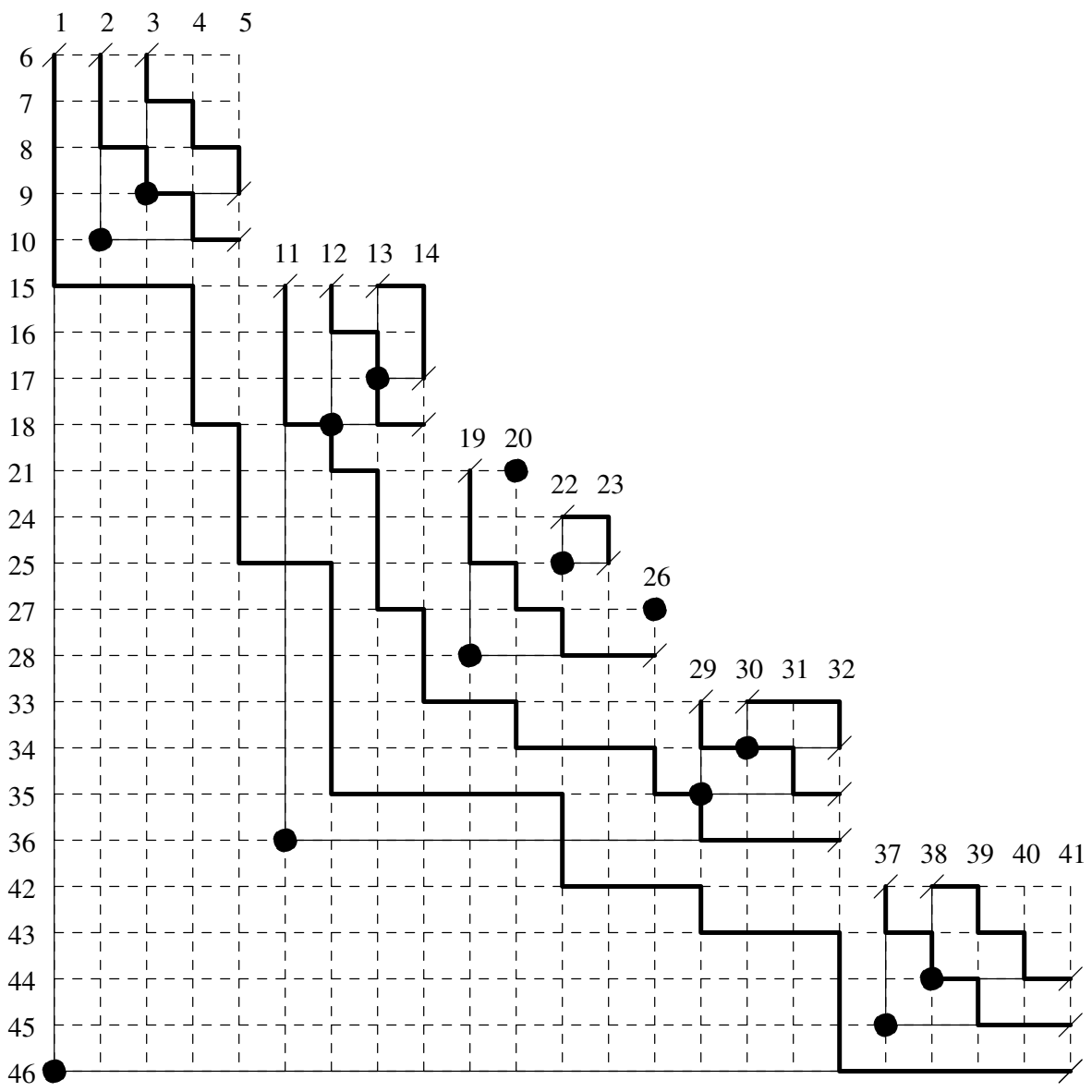

Figure 1. A symmetric tuple of nonintersecting lattice paths as in Example 6.1

line. Let $\beta$ (start) and $\beta$ (finish) denote respectively the points where the vertical line and the horizontal line meet the boundary. For example, $\beta$ (start $)=(15,11)$ and $\beta$ (finish) $=(36,32)$ for $\beta=(36,11)$; for $\beta=(21,20), \beta$ (start $)=\beta$ (finish) $=\beta$.

A lattice path between a pair of such points $\beta$ (start) and $\beta$ (finish) is a sequence $\alpha_{1}, \ldots, \alpha_{q}$ of elements of $\mathfrak{N}^{v}$ with $\alpha_{1}=\beta$ (start) and $\alpha_{q}=\beta$ (finish) such that for $1 \leq j \leq q-1$, if we let $\alpha_{j}=(r, c)$, then $\alpha_{j+1}$ is either $(R, c)$ or $(r, C)$, where $R$ is the least element of $[2 d] \backslash v$ that is bigger than $r$ and $C$ the least element of $v$ that is bigger than $c$. Note that if $\beta$ (start) $=(r, c)$ and $\beta$ (finish) $=(R, C)$, then $q$ equals

$$
|([2 d] \backslash v) \cap\{r, r+1, \ldots, R\}|+|v \cap\{c, c+1, \ldots, C\}|-1 .
$$

Write $\mathfrak{S}_{w}=\left\{\beta_{1}, \ldots, \beta_{p}\right\}$. By Proposition $5.7 \mathfrak{S}_{w}^{\#}=\mathfrak{S}_{w^{\#}}=\mathfrak{S}_{w}$; that is, $\mathfrak{S}_{w}$ is symmetric with respect to the anti-diagonal. Consider the set of all $p$-tuples of paths $\left(\Lambda_{1}, \ldots, \Lambda_{p}\right)$, where $\Lambda_{j}$ is a lattice path between $\beta_{j}$ (start) and $\beta_{j}$ (finish) such that

- No two $\Lambda_{j}$ intersect.

- If $\beta_{j}=\beta_{k}^{\#}$, then $\Lambda_{j}=\Lambda_{k}^{\#}$. 

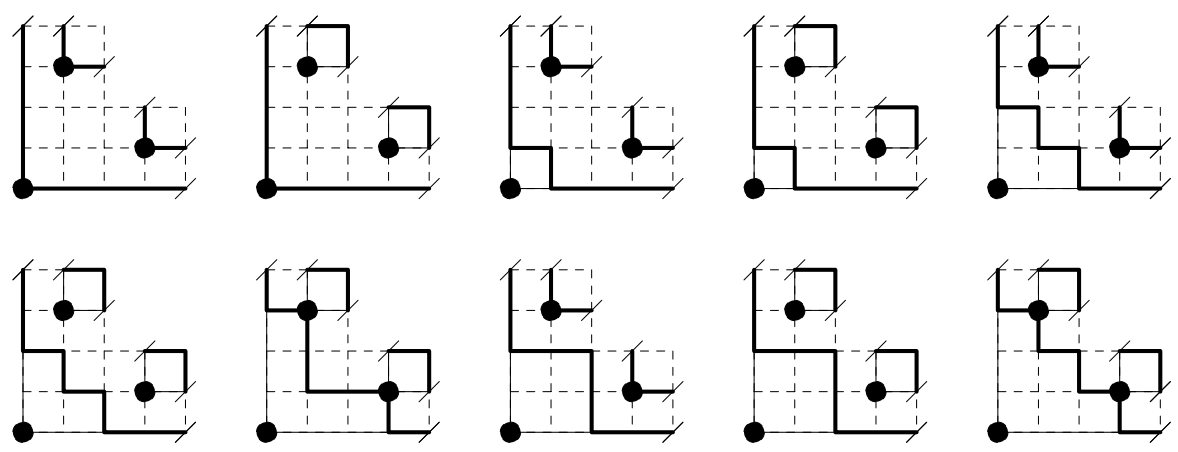

Figure 2. All the symmetric tuples of nonintersecting lattice paths as in Example 6.2

A particular such symmetric $p$-tuple is shown in Figure 1. The number of such symmetric $p$-tuples is the multiplicity of $X_{w}$ at the point $e^{v}$.

Example 6.2. Let us draw, in a simple case, the pictures of all possible symmetric tuples of nonintersecting lattice paths as defined in the above example. Let

$$
d=5, \quad v=(1,2,3,6,7) \quad \text { and } \quad w=(3,5,7,9,10)
$$

so that $\mathfrak{S}_{w}=\{(5,2),(9,6),(10,1)\}$. Figure 2 shows all the symmetric 3 -tuples of nonintersecting lattice paths. There are 10 of them, and thus the multiplicity in this case is 10 .

6.1. The Gröbner basis interpretation. Here we interpret Theorem 2.1 in terms of Gröbner basis; see Proposition 6.3 below. The special case of the proposition when $v$ is the identity coset (that is, $v=(1, \ldots, d)$ ) has been obtained by Conca 4 by different methods.

Fix elements $v, w$ in $I(d)$ with $v \leq w$. Recall from Section 3 that the ideal

$$
\left(f_{\mathfrak{w}}=p_{\mathfrak{w}} / p_{v} \mid \mathfrak{w} \text { admissible pair, } \mathfrak{t o p}(\mathfrak{w}) \not \leq w\right)
$$

in the polynomial ring $P:=k\left[X_{\beta} \mid \beta \in \mathfrak{R}^{v}\right]$ defines the tangent cone to the Schubert variety $X_{w}$ at the point $e^{v}$. We will identify a subset of these generators $f_{\mathfrak{w}}$ as being a Gröbner basis with respect to certain term orders for the ideal of the tangent cone. Observe that the $f_{\mathfrak{w}}$ are homogeneous polynomials in the variables $X_{\beta}$.

Let $\mathfrak{w}=(t, u)$ be an admissible pair and $\left(\theta, \theta^{\#}\right)$ the pair associated to $\mathfrak{w}$ as in Proposition 3.4. Let us call $\mathfrak{w}$ "good" (the scope of this terminology is intended to be limited to this section) if

- $v \leq u$ and $t \not \leq w$;

- $\mathfrak{S}_{\theta}=\mathfrak{S}_{\theta}^{\text {up }}$, that is, $r \leq c^{*}$ for $(r, c)$ in $\mathfrak{S}_{\theta}$, and the elements of $\mathfrak{S}_{\theta}$ form a $v$-chain-here $\mathfrak{S}_{\theta}$ is the monomial attached to $\theta$ as in Proposition 5.16

Suppose that $\mathfrak{w}$ is good. Then $\mathfrak{S}_{\theta}$ occurs up to sign as a term in the expression for $f_{\mathfrak{w}}=f_{\theta}$ as the determinant of a submatrix of the matrix of the form on page 5410 attached to $v$-here we are abusing notation and not distinguishing between a monomial in $\mathfrak{R}^{v}$ and the corresponding monomial in the variables $X_{\beta}, \beta$ in $\mathfrak{R}^{v}$.

\footnotetext{
${ }^{6}$ While it is true that in Section 5 we are using the notation of [14] where the symbols have a different meaning from what they do in Sections 24 the condition that $r \leq c^{*}$ for $(r, c)$ in $\mathfrak{S}_{\theta}$ gives us some license for sloppiness.
} 
Proposition 6.3. Fix elements $v \leq w$ of $I(d)$. Fix any term order on the monomials in the standard graded polynomial ring $P:=k\left[X_{\beta} \mid \beta \in \mathfrak{R}^{v}\right]$ such that, for any good admissible pair $\mathfrak{w}$, the initial term of $f_{\mathfrak{w}}$ is $\mathfrak{S}_{\theta}$. Then the $f_{\mathfrak{w}}$ as $\mathfrak{w}$ varies over all good admissible pairs form a Gröbner basis with respect to this term order for the ideal $\left(f_{\mathfrak{w}} \mid \mathfrak{w}\right.$ admissible pair, $\left.\mathfrak{t} \mathfrak{p}(\mathfrak{w}) \not \leq w\right)$ defining the tangent cone to the Schubert variety $X_{w}$ at the point $e^{v}$.

Proof. Denoting by $I$ the ideal $\left(f_{\mathfrak{w}} \mid \mathfrak{w}\right.$ admissible pair, $\left.\mathfrak{t o p}(\mathfrak{w}) \not \leq w\right)$ defining the tangent cone, by $\operatorname{in}(f)$ the initial term of a polynomial $f$ in the fixed term order, and by $\operatorname{in}(I)$ the ideal $(\operatorname{in}(f) \mid f \in I)$, we clearly have a graded surjection

$$
P /\left(\operatorname{in}\left(f_{\mathfrak{w}}\right) \mid \mathfrak{w} \text { good admissible pair, } \mathfrak{t o p}(\mathfrak{w}) \not \leq w\right) \rightarrow P / \text { in }(I) .
$$

The assertion is that this map is an isomorphism. To prove this, it is enough to show that the Hilbert function of the quotient ring dominates that of the ring on the left.

The Hilbert function of $P / \operatorname{in}(I)$ is the same as that of the tangent cone $P / I$, and so by Theorem 2.1 its value at a positive integer $m$ is the cardinality of the set $S_{w}^{v}(m)$ of $w$-dominated monomials in $\mathfrak{R}^{v}$ of degree $m$. It therefore suffices to observe that a monomial in $\mathfrak{R}^{v}$ that is not $w$-dominated belongs to the ideal $\left(\operatorname{in}\left(f_{\mathfrak{w}}\right) \mid \mathfrak{w}\right.$ good admissible pair, $\left.\mathfrak{t o p}(\mathfrak{w}) \not \leq w\right)$. Given such a monomial, choose a $v$-chain $\beta_{1}>\ldots>\beta_{t}$ in it such that $w \nsupseteq s_{\beta_{1}} \cdots s_{\beta_{t}} v$. Applying the lemma below to the this $v$-chain, we get a good admissible pair $\mathfrak{w}$ such that the initial term of $f_{\mathfrak{w}}$ in the fixed term order is $\left\{\beta_{1}, \ldots, \beta_{t}\right\}$.

Lemma 6.4. Fix elements $v \leq w$ of $I(d)$. Let $\beta_{1}>\ldots>\beta_{r}$ be a $v$-chain of elements in $\mathfrak{N}^{v}$ such that $w \nsupseteq s_{\beta_{1}} \cdots s_{\beta_{r}} v$. The element of $\mathrm{SM}^{v, v}$ that corresponds under the bijection of Proposition 4.1 to the element $\left\{\beta_{1}, \ldots, \beta_{r}\right\}$ of $T^{v}$ consists of a single admissible pair $\mathfrak{w}=(t, u)$ which is good and for which $\mathfrak{S}_{\theta}=\left\{\beta_{1}, \ldots, \beta_{r}\right\}$.

Proof. We follow the notation of the statement and proof of Proposition 4.1. Set $\mathfrak{S}=\left\{\beta_{1}, \ldots, \beta_{r}\right\} \cup\left\{\beta_{1}^{\#}, \ldots, \beta_{r}^{\#}\right\}, \pi(\mathfrak{S})=\left(t, \mathfrak{S}^{\prime}\right)$, and $\pi\left(\mathfrak{S}^{\prime}\right)=(u, \mathfrak{T})$. We know from that proposition that $(t, u)$ is an admissible pair and that $v \leq u$. Since the bijection $\mathrm{SM}^{v, v} \simeq T^{v}$ respects domination, and $s_{\beta_{1}} \cdots s_{\beta_{r}} v \not \leq w$ by hypothesis, it follows that $t \not \leq w$. It remains to be seen that $\mathfrak{T}$ is the empty monomial and that $\mathfrak{S}_{\theta}=\left\{\beta_{1}, \ldots, \beta_{r}\right\}$. These will follow from explicit computation which we now perform.

Set $\beta_{j}=\left(r_{j}, c_{j}\right)$; then $\beta_{j}^{\#}=\left(c_{j}^{*}, r_{j}^{*}\right)$. Since $\beta_{j} \in \mathfrak{N}^{v}$, we have $c_{j} \leq r_{j}^{*}$ and $c_{j}<r_{j}$, and so $\left\{c_{1}, \ldots, c_{t}\right\} \subseteq[d]$.

Consider the two-step partitioning of $\mathfrak{S}$ into the $\mathfrak{S}_{j}$ and the $\mathfrak{S}_{j}$ into blocks. It is readily seen that $\mathfrak{S}_{j}=\left\{\beta_{j}, \beta_{j}^{\#}\right\}$. Let $s$ be the largest integer, $0 \leq s \leq r$, such that $r_{s} \geq r_{s}^{*}$; set $s=0$ if $r_{1}<r_{1}^{*}$. In other words, $s$ is the least integer, $0 \leq s \leq t$, such that $\left\{r_{s+1}, \ldots, r_{t}\right\} \subseteq[d]$. For $1 \leq j \leq s, \mathfrak{S}_{j}$ is a single block. For $s<j \leq t$, the block decomposition of $\mathfrak{S}_{j}$ is $\left\{\beta_{j}\right\} \cup\left\{\beta_{j}^{\#}\right\}$.

The following expressions for $\mathfrak{S}^{\prime}, u, t$, and $\mathfrak{T}$ follow readily from the definition of the map $\pi$ :

$$
\mathfrak{S}^{\prime}=\left\{\left(r_{1}, r_{1}^{*}\right), \ldots,\left(r_{t}, r_{t}^{*}\right)\right\} \quad \text { and } \quad u=\left(v \cup\left\{r_{1}, \ldots, r_{s}\right\}\right) \backslash\left(\left\{r_{1}^{*}, \ldots, r_{s}^{*}\right\}\right)
$$

and

$$
t=\left(v \cup\left\{c_{1}^{*}, \ldots, c_{t}^{*}\right\} \cup\left\{r_{s+1}, \ldots, r_{t}\right\}\right) \backslash\left(\left\{c_{1}, \ldots, c_{t}\right\} \cup\left\{r_{s+1}^{*}, \ldots, r_{t}^{*}\right\}\right),
$$


and $\mathfrak{T}$ is the empty monomial. Now, upon letting $[d]^{\mathrm{c}}:=\{d+1, \ldots, 2 d\}$, put

$$
\theta:=(t \cap[d]) \cup\left(u \cap[d]^{c}\right)=\left(v \cup\left\{r_{1}, \ldots, r_{t}\right\}\right) \backslash\left\{c_{1}, \ldots, c_{t}\right\}
$$

and

$$
\theta^{\#}:=(u \cap[d]) \cup\left(t \cap[d]^{c}\right)=\left(v \cup\left\{c_{1}^{*}, \ldots, c_{t}^{*}\right\}\right) \backslash\left\{r_{1}^{*}, \ldots, r_{t}^{*}\right\} .
$$

Immediately from the definition of $\mathfrak{S}_{\theta}$, we have

$$
\mathfrak{S}_{\theta}=\left\{\left(r_{1}, c_{1}\right), \ldots,\left(r_{t}, c_{t}\right)\right\} \quad \text { and } \quad \mathfrak{S}_{\theta \#}=\left\{\left(c_{1}^{*}, r_{1}^{*}\right), \ldots,\left(c_{t}^{*}, r_{t}^{*}\right)\right\} .
$$

Thus the lemma is proved.

We finish by listing some term orders that satisfy the requirement of Proposition 6.3. Fix notation and terminology as in $\S 15.2$ of [7]. Defined below are eight total orders $>_{1}$ through $>_{8}$ on the elements of $\mathfrak{N}^{v}$. Let $>_{j}$ denote also any total order on $\mathfrak{R}^{v}$ that refines the total order $>_{j}$ on $\mathfrak{N}^{v}$. The homogeneous lexicographic orders induced by $>_{1},>_{2},>_{7},>_{8}$ and the reverse lexicographic orders induced by $>_{4},>_{6}$ satisfy the requirement of Proposition 6.3. The reverse lexicographic orders induced by $>_{3},>_{5}$ do not satisfy the requirement.

The total orders $>_{1}$ through $>_{8}$ on $\mathfrak{N}^{v}$ are as follows: for $(r, c)$ in $\mathfrak{N}^{v}$ and $(a, b)$ in $\mathfrak{R}^{v} \backslash \mathfrak{N}^{v}$

(1) $(r, c)>_{1}\left(r^{\prime}, c^{\prime}\right)$ if either (a) $r<r^{\prime}$ or (b) $r=r^{\prime}$ and $c>c^{\prime}$.

(2) $(r, c)>_{2}\left(r^{\prime}, c^{\prime}\right)$ if either (a) $c>c^{\prime}$ or (b) $c=c^{\prime}$ and $r<r^{\prime}$.

(3) $(r, c)>_{3}\left(r^{\prime}, c^{\prime}\right)$ if either (a) $r<r^{\prime}$ or (b) $r=r^{\prime}$ and $c<c^{\prime}$.

(4) $(r, c)>_{4}\left(r^{\prime}, c^{\prime}\right)$ if either (a) $c>c^{\prime}$ or (b) $c=c^{\prime}$ and $r>r^{\prime}$.

(5) $(r, c)>_{5}\left(r^{\prime}, c^{\prime}\right)$ if either (a) $c<c^{\prime}$ or (b) $c=c^{\prime}$ and $r<r^{\prime}$.

(6) $(r, c)>_{6}\left(r^{\prime}, c^{\prime}\right)$ if either (a) $r>r^{\prime}$ or (b) $r=r^{\prime}$ and $c>c^{\prime}$.

(7) $(r, c)>_{7}\left(r^{\prime}, c^{\prime}\right)$ if either (a) $c<c^{\prime}$ or (b) $c=c^{\prime}$ and $r>r^{\prime}$.

(8) $(r, c)>_{8}\left(r^{\prime}, c^{\prime}\right)$ if either (a) $r>r^{\prime}$ or (b) $r=r^{\prime}$ and $c<c^{\prime}$.

\section{ACKNOWLEDGMENTS}

A part of this work was done when the first-named author visited the Institute of Mathematical Sciences, Chennai, during March 2003, and Purdue University, West Lafayette, during Spring 2004. He would like to thank both these institutions for their hospitality and support. Also, he gratefully acknowledges the travel support from the Commission on Development and Exchanges of the International Mathematical Union for his visit to Purdue.

\section{REFERENCES}

1. S. S. Abhyankar, Enumerative combinatorics of Young tableaux, Marcel Dekker, New York, 1988. MR0926272 (89e:05011)

2. M. Brion and P. Polo, Generic singularities of certain Schubert varieties, Math. Z. 231 (1999), 301-324. MR1703350 (2000f:14078)

3. S. Billey and V. Lakshmibai, Singular Loci of Schubert Varieties, Progress in Math., Vol. 182, Birkhäuser, Boston, 2000. MR.1782635 (2001j:14065)

4. A. Conca, Gröbner bases of ideals of minors of a symmetric matrix, J. Algebra 166 (1994), 406-421. MR:1279266 (95g:13012)

5. A. Conca and J. Herzog, On the Hilbert function of determinantal rings and their canonical module, Proc. Amer. Math. Soc. 122 (1994), 677-681. MR1213858 (95a:13016)

6. C. De Concini, Symplectic Standard Tableaux, Adv. Math. 34 (1979), 1-27. MR0547837 (80m:14036)

7. D. Eisenbud, Commutative Algebra with a View Toward Algebraic Geometry, Grad. Texts in Math., Vol. 150, Springer-Verlag, New York, 1995. MR.1322960 (97a:13001) 
8. W. Fulton and P. Pragacz, Schubert Varieties and Degeneracy Loci, Lect. Notes in Math., Vol. 1689, Springer-Verlag, Berlin, Heidelberg, 1995. MR.1639468 (99m:14092)

9. S. R. Ghorpade, Abhyankar's work on Young tableaux and some recent developments, Algebraic Geometry and its Applications (West Lafayette, IN, 1990), pp. 235-265, Springer-Verlag, New York, 1994. MR.1272033(95h:05148)

10. S. R. Ghorpade, Young bitableaux, lattice paths and Hilbert functions, J. Statist. Plann. Inference 54 (1996), 55-60. MR1406731 (98c:05165)

11. S. R. Ghorpade and C. Krattenthaler, The Hilbert series of Pfaffian rings, Algebra, Arithmetic and Geometry with Applications (West Lafayette, IN, 2000), pp. 337-356, Springer, Berlin, 2004. MR2037100 (2005c:13010)

12. J. Herzog and N. V. Trung, Gröbner bases and multiplicity of determinantal and pfaffian ideals, Adv. Math. 96 (1992), 1-37. MR1185786 (94a:13012)

13. A. Knutson and E. Miller, Gröbner Geometry of Schubert Polynomials, Ann. Math. (2) 161 (2005), 1245-1318. MR2180402

14. V. Kodiyalam and K. N. Raghavan, Hilbert functions of points on Schubert varieties in Grassmannians, J. Algebra 270 (2003), 28-54. MR2015929 (2005d:14067)

15. C. Krattenthaler, On multiplicities of points on Schubert varieties in Grassmannians, Sem. Lothar. Combin. 45 (2000/01), Art.B45c, 11pp (electronic). MR1817336 (2002c:14080)

16. C. Krattenthaler, On multiplicities of points on Schubert varieties in Grassmannians II, J. Algebraic Combin. 22 (2005), 273-288. MR2181366

17. V. Kreiman, Monomial Bases and Applications for Richardson and Schubert Varieties in Ordinary and Affine Grassmannians, Thesis, Northeastern University, 2003.

18. V. Kreiman and V. Lakshmibai, Multiplicities of Singular Points of Schubert Varieties of Grassmannians, Algebra, Arithmetic and Geometry with Applications (West Lafayette, IN, 2000), pp. 553-563, Springer, Berlin, 2004. MR2037109 (2005c:14060)

19. V. Kreiman and V. Lakshmibai, Richardson Varieties in the Grassmannians, Contributions to Automorphic Forms, Geometry and Number Theory (Baltimore, MD, 2002), pp. 573-597, Johns Hopkins Univ. Press, Baltimore, MD, 2004. MR2058620 (2005c:14059)

20. V. Lakshmibai, C. Musili and C. S. Seshadri, Geometry of $G / P$ - IV, Proc. Indian Acad. Sci. Sect. A Math. Sci. 88 (1979), 279-362. MR0553746 (81g:14023d)

21. V. Lakshmibai and C. S. Seshadri, Geometry of $G / P$ - II, Proc. Indian Acad. Sci. Sect. A 87 (1978), 1-54. MR0490244(81g:14023b)

22. V. Lakshmibai and J. Weyman, Multiplicities of points on a Schubert variety in a minuscule $G / P$, Adv. Math. 84 (1990), 179-208. MR1080976 (92a:14058)

23. P. Littelmann, A Littlewood-Richardson rule for symmetrizable Kac-Moody algebras, Invent. Math. 116 (1994), 329-346. MR1253196 (95f:17023)

24. P. Littelmann, Paths and root operators in representation theory, Ann. of Math. (2) 142 (1995), 499-525. MR1356780(96m:17011)

25. L. Manivel, Fonctions Symétriques, Polynômes de Schubert et Lieux de Dégénérescence, Cours Spécialisés No. 3, Soc. Math. de France, Paris, 1998. MR1638048 (99k:05159)

26. J. Rosenthal and A. Zelevinsky, Multiplicities of points on Schubert varieties in Grassmannians, J. Algebraic Combin. 13 (2001), 213-218. MR1826954(2002d:14089)

Department of Mathematics, Indian Institute of Technology Bombay, Powai, Mumbai 400076 , INDIA

E-mail address: srg@math.iitb.ac.in

Institute of Mathematical Sciences, C.I.T. Campus, Taramani, Chennai 600 113, India

E-mail address: knr@imsc.ernet.in 